\title{
Neue Ergebnisse auf dem Gebiet der Bisbenzylisochinolin-Alkaloide
}

\section{Recent Advances in Bisbenzylisoquinoline Alkaloids}

\author{
Peter Pachaly \\ Pharmazeutisches Institut der Universität Bonn, Kreuzbergweg 26, D-5300 Bonn 1, Federal Republic of Germany
}

Received: December 6, 1988

Abstract
This review presents results of the
chemistry and pharmacology of the bisben-
zylisoquinoline alkaloids which are reported from 1986
through 1988.
Key words
BBI-alkaloids, structure determination,
pharmacological activity, cell culture, synthesis, bio-
synthesis.

\section{Einleitung}

Die sich durch große strukturelle Variationsbreite auszeichnenden Bisbenzylisochinolin-Alkaloide (BBI-AJkaloide) wurden in den vergangenen Jahren in mehreren ausführlichen tabellarischen Übersichtsartikeln [Guha et al. 1979 (1), Schiff Jr. 1983 (2) und 1987 (3)] dargestellt, wobei die Literatur bis 1985 berücksichtigend 271 Vertreter dieser Alkaloidklasse erfaßt wurden. Weitere zum Teil sich auf einzelne Pflanzengattungen beschränkende aktuelle Übersichten finden sich in der Literatur (5, $6,157,158)$. Die erst durch die modernen spektroskopischen Methoden möglichen Erfolge bei der Strukturaufklärung der oft nur mit wenigen mg vorliegenden Naturstoffe haben auch die Zahl der bekannten BBI-Alkaloide in den letzten Jahren gewaltig ansteigen lassen, so daß seit 1985 bis November 1988 bereits weitere 105 neue BBI-Alkaloide bekannt geworden sind, die in diesem Übersichtsartikel beschrieben werden sollen.

Die Strukturformeln wurden nach den von Shamma et al. $(4,159)$ für die BBI-Alkaloide vorgeschlagenen Konventionen abgebildet und die Typen nach dem Ort der Sauerstoffsubstitution gekennzeichnet. Die Nummerierung der bekannten BBI-Alkaloide in den bisherigen Übersichten $(1,2,3)$ wurde beibehalten und durch eckige Klammern [] gekennzeichnet.

Obwohl die vollständige Wiedergabe aller zum Strukturbeweis herangezogenen Daten für den schnellen Vergleich eigener Meßergebnisse mit den Literaturda- ten besonders wichtig erscheint, kann hier leider aus Platzgründen nur auf die Originalliteratur verwiesen werden. Secobisbenzylisochinolin-Alkaloide wurden ebenfalls hier aufgenommen, da sie als Metabolisierungsprodukte von BBI-Alkaloiden anzusehen sind.

BBI-Alkaloid-haltige Drogen spielen in der Volksmedizin der tropischen und subtropischen Länder eine große Rolle. Die mit einzelnen, daraus isolierten Alkaloiden vermehrt durchgeführten pharmakologischen Untersuchungen ergaben erstaunlich vielfältige Aktivitäten dieser Verbindungen, die damit auch eine Wirkung der Drogen in wachsendem Umfang zu bestätigen scheinen. Obwohl mit den meisten der hier vorgestellten neuen BBIAlkaloiden aber noch kein pharmakologisches Screening durchgeführt werden konnte, sind alle Vertreter dieser Alkaloidklasse hierfür interessant. Ein großes Hindernis für eine ausführliche pharmakologische Untersuchung ist aber nach wie vor für die meisten Alkaloide dieser Stoffklasse die schwierige oder gar unmögliche Beschaffung genügend großer Mengen. Hier könnten sich in der Zukunft die denkbare Gewinnung durch Zellkulturen oder die (partial)synthetische Abwandlung von BBI-Alkaloiden, die in ausreichender Menge zugänglich sind (z. B. Tetrandrin), als hilfreich erweisen.

\section{Analytik}

Bei der Strukturaufklärung der zahlreichen BBI-Alkaloide haben sich die Fortschritte der letzten Jahre in der routinemäßigen Messung von ${ }^{1} \mathrm{H}$ - und ${ }^{13} \mathrm{C}$-NMRSpektren insbesondere mit den Möglichkeiten von hochauflösenden 2D-Meßtechniken ebenso wie NOE-Spektren (z. B. 8, 9, 12, 22, 23, 29, 166) als außerordentlich nützlich erwiesen. Bei den makrocyclischen BBI läßt sich innerhalb der einzelnen Gruppen aus den NMR-Spektren zusammen mit der spezifischen Drehung auf die absolute Konfiguration schließen: $S, S$ - bzw. $R, R$-Verbindungen einerseits und $S, R$ - bzw. $R, S$-BBI andererseits unterscheiden sich erwartungsgemäß in den ${ }^{1} \mathrm{H}$-NMR-Spektren im Bereich der aromatischen Protonensignale in charakteristischer Weise, was in zahlreichen Beispielen in einer Übersicht (159) belegt ist, aber auch von anderen Arbeitsgruppen für weitere Beispiele beobachtet wurde (z. B. 25, 27). Hiermit wurde die Aussagekraft der spez. Drehung allein bestätigt, die innerhalb der einzelnen Gruppen der makrocyclischen BBIAlkaloide bereits die Konfigurationszuordnung der beiden 


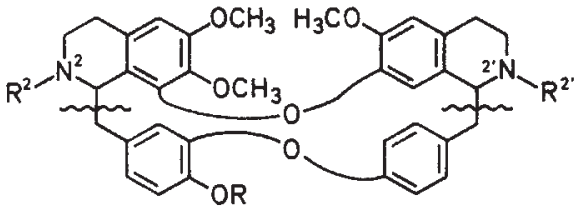

Abb. 1 Photoxidative Spaltung von BBI-Alkaloiden (43).

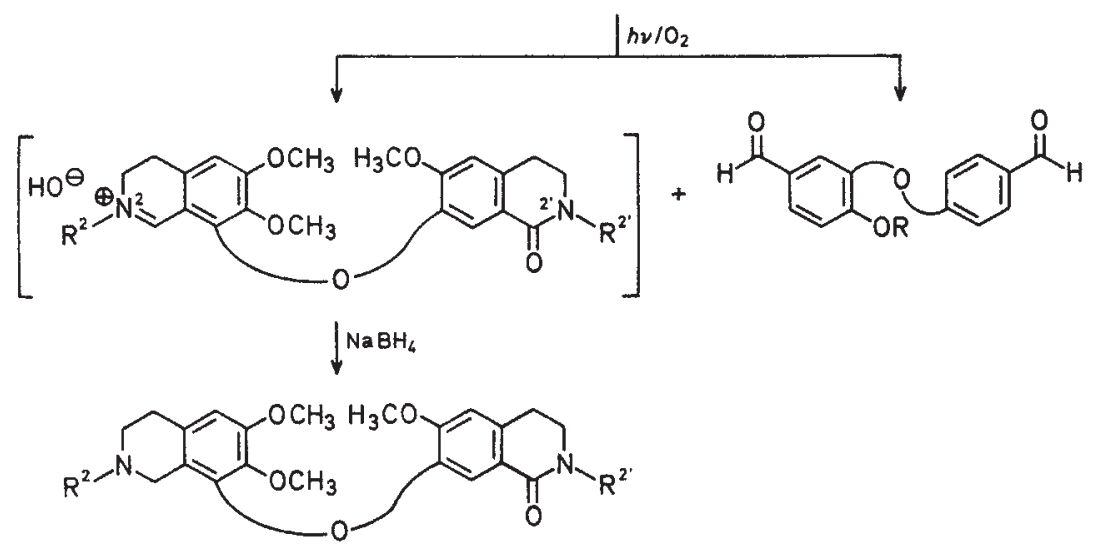

Chiralitätszentren am C-1 und C-1' ermöglicht (165). Als ebenso nützlich für die Konfigurationsbestimmung haben sich auch CD-Messungen gezeigt $(7,9,11,29,166)$.

Hochaufgelöste MS ermöglichen in der Regel die schnelle Zuordnung der BBI-Alkaloide zum jeweiligen Grundtyp, wobei die genaue Messung der Fragmentionen bereits eine Entscheidung über das generelle Substitutionsmuster zuläßt. Schwierigkeiten bei der Erkennung der Molekülionen (besonders beim Dauricin-Typ) wurden durch Einsatz weicher Ionisationsmethoden erfolgreich überwunden, hierbei sind besonders $(M+1)$-Fragmente charakteristisch $(7,9,10,49)$. Die Kombination von GC/MS wurde erfolgreich zur Identifizierung der Alkaloide von Stephania cepharantha eingesetzt (38).

Neben den spektroskopischen Methoden werden bei der Strukturaufklärung nach wie vor Derivate durch $O$-bzw. $N$-Methylierung (Diazomethan bzw. $\mathrm{NaBH}_{4}$ ' Formaldehyd) hergestellt, weil dabei sehr oft bekannte BBIAlkaloide erhalten werden. Als Ergänzung zum klassischen reduktiven $\mathrm{BBI}-\mathrm{Abbau}$ mit Natrium in flüssigem Ammoniak wurde kürzlich eine interessante photooxidative Alternativ-Methode vorgestellt (43) (Abb. 1), bei der bevorzugt, (ähnlich wie bei der MS von makrocyclischen BBI-Alkaloiden) zwischen Isochinolin- und Benzylschwanzteil gespalten wird.

Röntgenstrukturanalyse wird bei BBI-Alkaloiden selten eingesetzt, wohl auch, weil die Verbindungen meist amorph anfallen. Ein aktuelles Beispiel ist die Kristallstrukturanalyse von Cycleanin aus Cissampelos pareira L. (77). Ein Charakteristikum der makrocyclischen BBI-Alkaloide ist die immer wieder beobachtete Tendenz, Lösungsmittel hartnäckig einzuschließen, was die Elementaranalysen ebenso wie die sehr unterschiedlichen Schmelzpunktangaben für gleiche Verbindungen belegen. Oft ist das zuletzt benutzte Lösungsmittel in den NMR- und MS-Spektren eindeutig nachzuweisen.
Einfache Gehaltsbestimmungen von BBIAlkaloiden in organischen Lösungsmitteln durch wasserfreie potentiometrische Titration wurden z. B. für Berbamin $(73,74)$ beschrieben. Eine Spektralphotometrische Bestimmung nach Bildung eines lonenkomplexes mit Methylorange gelang mit Tubocurarin (78); Trilobin wurde nach Extraktion aus dem Blutplasma mit HPLC (153), aber auch durch quantitative DC bei $265 \mathrm{~nm}$ bestimmt (75).

\section{Neue BBI-Alkaloide}

Die Strukturen der neuen BBI-Alkaloide werden in den $\mathrm{Abb} .2$ bis 20 vorgestellt. Obwohl die meisten dieser Alkaloide sich lediglich als Variationen und Isomere bekannter Grundtypen erwiesen, sind auch einige völlig neue BBI-Strukturen gefunden worden. Als Erfolge moderner Trennmethoden sind vermehrt stärker polare Verbindungen wie $N$-Oxide, $N$-Norverbindungen und mehrwertige Phenole zu verzeichnen. Alle asymmetrischen KopfKopf/Schwanz-Schwanz verknüpften Verbindungen gehören dem Biscoclaurintyp an, lediglich zwei Beispiele sind dem Bisreticulin-Typ zuzurechnen [Efatin (97) und Ambrinin (98)]. Insgesamt wurden nur drei neue Kopf-Schwanz verknüpfte BBI-Alkaloide in den vergangenen 3 Jahren beschrieben.

Bei den Verbindungen vom Dauricin-Typ (Abb. 2) sind als besondere Strukturvariationen die relativ seltenen phenolischen $\mathrm{OH}-\mathrm{Gruppen}$ in 6-und 6'-Stellung zu verzeichnen. Die $N$-Oxide von $O$-Methyldauricin (6 und 7) (7) wurden lediglich als Gemisch charakterisiert, während für Neothalibrin-2' $\alpha-N$-oxid (12) die Konfiguration am $2^{\prime}-\mathrm{N}$ durch NOE-Experimente bewiesen wurde, aber auch bereits an der ${ }^{1} \mathrm{H}-\mathrm{NMR}$-Tieffeldverschiebung des $2^{\prime}-N$-Methylsignals nach 3,24 ppm erkannt wurde (161).

Die absolute Konfiguration der Chiralitätszentren an $\mathrm{C}-1$ und $\mathrm{C}-1$ ' wurde für die Beispiele 1-7 durch klassischen Abbau und durch Umwandlung in $O$-Methyldauricin bewiesen, während die $R, R$-Konfiguration von 2Norberbamunin (8) ebenso wie bei den Beispielen 13, 14 und 15 aus den CD-Messungen abgeleitet wurde. 


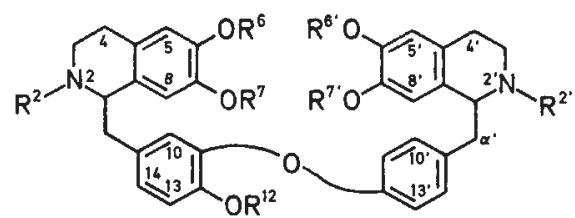

Abb. 2 Neue BBI-Alkaloide vom Dauricin-Typ - Typ I: 6,7,11*,12 - 6,7,12* (1).

\begin{tabular}{|c|c|c|c|c|c|c|c|c|c|c|}
\hline Verbdg. & abs. Konfg. & Name & $\mathrm{R}^{2}$ & $\mathrm{R}^{6}$ & $\mathrm{R}^{7}$ & $\mathbf{R}^{12}$ & $\mathrm{R}^{2^{\prime}}$ & $\mathrm{R}^{6^{\prime}}$ & $\mathrm{R}^{7^{\prime}}$ & Lit. \\
\hline 2 & $1 R, 1^{\prime} R$ & Popisidin & $\mathrm{CH}_{3}$ & $\mathrm{CH}_{3}$ & $\mathrm{CH}_{3}$ & $\mathrm{CH}_{3}$ & $\mathrm{CH}_{3}$ & $\mathrm{H}$ & $\mathrm{CH}_{3}$ & 7 \\
\hline 5 & $1 R, 1^{\prime} R$ & Popisonin & $\mathrm{CH}_{3}$ & $\mathrm{H}$ & $\mathrm{CH}_{3}$ & $\mathrm{CH}_{3}$ & $\mathrm{CH}_{3}$ & $\mathrm{H}^{\circ}$ & $\mathrm{CH}_{3}$ & 7 \\
\hline 6 & $1 R, I^{\prime} R$ & O-Methyldauricin-2- $\mathrm{N}$-oxid & $\mathrm{CH}_{3} / \mathrm{N}$-Oxid & $\mathrm{CH}_{3}$ & $\mathrm{CH}_{3}$ & $\mathrm{CH}_{3}$ & $\mathrm{CH}_{3}$ & $\mathrm{CH}_{3}$ & $\mathrm{CH}_{3}$ & 7 \\
\hline 7 & $I R, I^{\prime} R$ & O-Methyldauricin-2'-N-oxid & $\mathrm{CH}_{3}$ & $\mathrm{CH}_{3}$ & $\mathrm{CH}_{3}$ & $\mathrm{CH}_{3}$ & $\mathrm{CH}_{3} / \mathrm{N}$-Oxid & $\mathrm{CH}_{3}$ & $\mathrm{CH}_{3}$ & 7 \\
\hline 10 & $1 R, 1^{\prime} R$ & 2-N-Methyllindoldhamin & $\mathrm{CH}_{3}$ & $\mathrm{CH}_{3}$ & $\mathrm{H}$ & $H$ & $H$ & $\mathrm{CH}_{3}$ & $\mathrm{H}$ & 20 \\
\hline 11 & $1 R, 1^{\prime} R$ & 2'-N-Methyllindoldhamin & $\mathrm{H}$ & $\mathrm{CH}_{3}$ & $H$ & $H$ & $\mathrm{CH}_{3}$ & $\mathrm{CH}_{3}$ & $H$ & 20 \\
\hline 12 & $1 S, 1^{\prime} R$ & Neothalibrin- $2^{\prime}-\alpha-N$-oxid & $\mathrm{CH}_{3}$ & $\mathrm{CH}_{3}$ & $\mathrm{H}$ & $\mathrm{CH}_{3}$ & $\mathrm{CH}_{3} / \alpha-N-0 x i d$ & $\mathrm{CH}_{3}$ & $\mathrm{CH}_{3}$ & 161 \\
\hline 13 & $1 R, 1^{\prime} R$ & Geraldoamin & $\mathrm{CH}_{3}$ & $\mathrm{CH}_{3}$ & $\mathrm{H}$ & $\mathrm{CH}_{3}$ & $\mathrm{H}$ & $\mathrm{CH}_{3}$ & $\mathrm{CH}_{3}$ & 162 \\
\hline 14 & $1 R, 1^{\prime} R$ & Pedroamin & $\mathrm{CH}_{3}$ & $\mathrm{CH}_{3}$ & $\mathrm{H}$ & $\mathrm{H}$ & $\mathrm{H}$ & $\mathrm{CH}_{3}$ & $H$ & 162 \\
\hline 15 & $1 R, 1^{\prime} R$ & Pampulhamin & $\mathrm{CH}_{3}$ & $\mathrm{CH}_{3}$ & $H$ & $\mathrm{H}$ & $\mathrm{H}$ & $\mathrm{CH}_{3}$ & $\mathrm{CH}_{3}$ & 162 \\
\hline
\end{tabular}

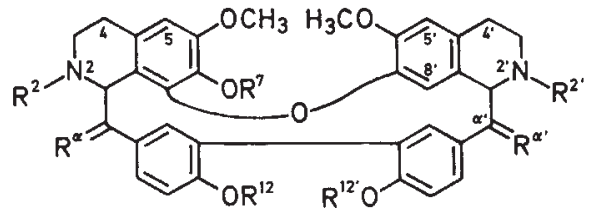

Abb. 3 Neue BBI-Alkaloide vom Rodiasin-Typ - Typ IV: 6,7,8*,12-6,7*,12 (11-11) (1).

\begin{tabular}{|c|c|c|c|c|c|c|c|c|c|c|}
\hline Verbdg. & abs. Konfg. & Name & $\mathrm{R}^{2}$ & $R^{7}$ & $\mathrm{R}^{12}$ & $R^{\alpha}$ & $\mathrm{R}^{2^{\prime}}$ & $\mathrm{R}^{12^{\prime}}$ & $\mathrm{R}^{\alpha}$ & Lit. \\
\hline
\end{tabular}

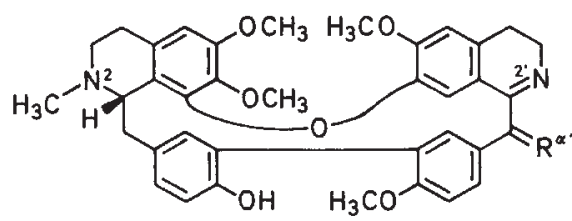

\begin{tabular}{llll}
\hline 25 & $1 S(+)$ & Guattamin $\left(\mathrm{R}-\alpha^{\prime}=\mathrm{H}_{2}\right)$ & 166 \\
26 & $1 S(+)$ & Guattaminon $\left(\mathrm{R}-\alpha^{\prime}=0\right)$ & 166 \\
\hline
\end{tabular}

Beim Rodiasin-Typ (Abb. 3) fallen einige $\alpha$ bzw. $\alpha^{\prime}$-Oxo-Derivate auf, die aus der Annonacee Pseudoxandra aff. lucida isoliert wurden. Bis auf Tilitriandrin (16), das hier als biogenetische Vorstufe der Tiliacora-Alkaloide in der Menispermacee Tiliacora triandra gelten muß, sind alle anderen Beispiele aus Annonaceen isoliert worden. Als neuartige Variante des Rodiasin-Typs ist das Medelline (27) aufzufassen, das ebenfalls aus der Rinde von Pseudoxandra aff. lucida erhalten wurde. Es ist das erste BBI-Beispiel mit einer Methylendioxybrücke zwischen den beiden Isochinolinteilen.

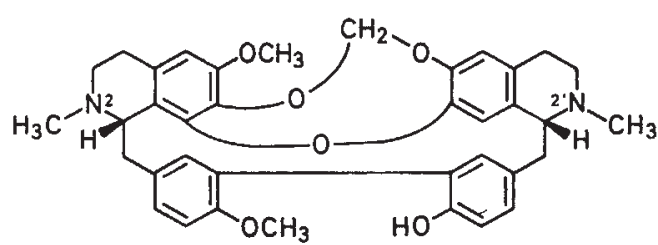

Abb. 4 1S, 1'R Medellin (Verbdg. 27) (8) - Rodiasin-Typ; Typ IV: $6,7,8^{*}, 12-6,7^{*}, 12,(11-11)(1)$. 


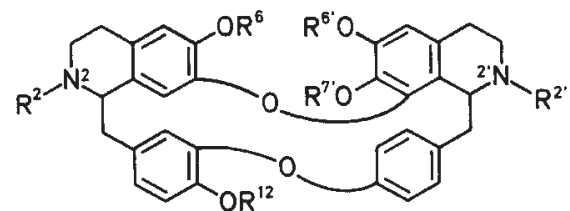

Abb. 5 Neue BBI-Alkaloide vom Oxyacanthin-Typ - Typ VI: 6,7*11\#,12-6,7,8*,12\# (1)

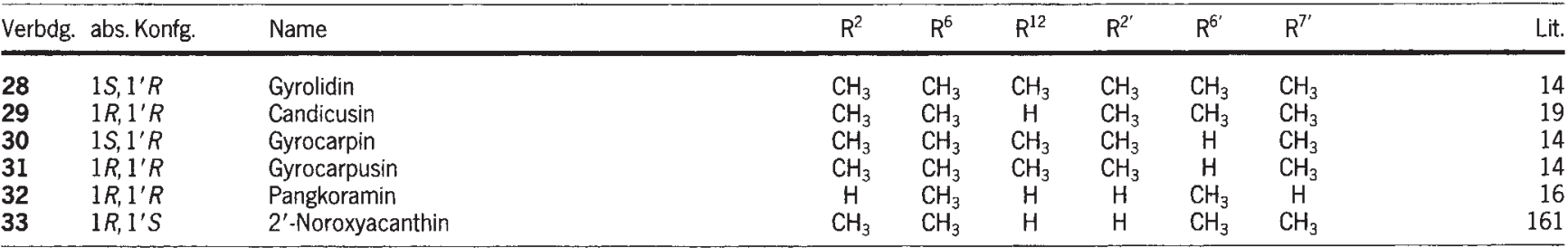

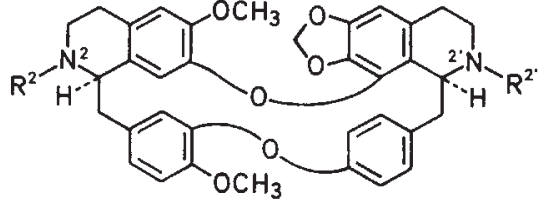

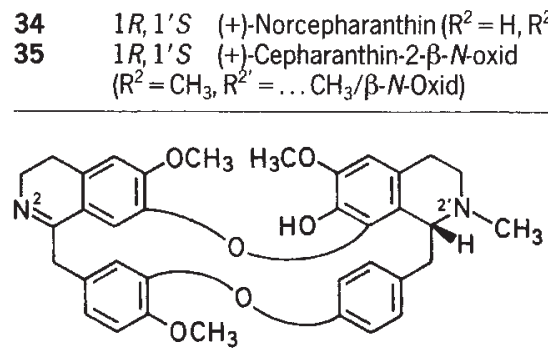

$38 \quad 1^{\prime} R \quad(-)-1,2-$ Dehydro-2-nor-limacusin

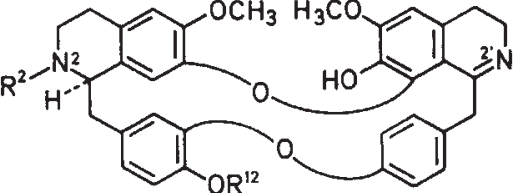

$361 R \quad(+)^{\prime}, 4^{\prime}$-Dihydro-stephasubin $\left\langle\mathrm{R}^{2}=\mathrm{CH}_{3}, \mathrm{R}^{12}=\mathrm{CH}_{3}\right)$

$37 \quad 1 R \quad\left(+\right.$-Pangkorimin $\left(\mathrm{R}^{2}=\mathrm{H}, \mathrm{R}^{12}=\mathrm{H}\right)$

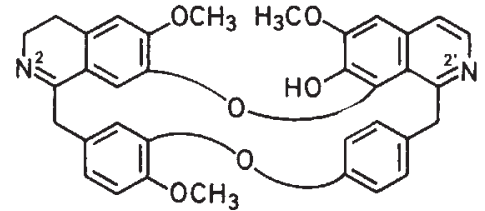

(17) $39 \quad$ Stephasubimin<smiles>[R20]Oc1ccc2cc1COCc1ccc(cc1)C[C@H]1c3cc(c(O)cc3CCN1[R2])Cc1nccc3cc(OC)c(O)c(c13)C2</smiles>

Abb. 7 Neue BBI-Alkaloide vom Oxyacanthin-Typ - Typ VI:6,7*,11\#,12-6,7,8*,12\# (1).

\begin{tabular}{lllllr}
\hline Verbdg, abs.Konfg. & Name & $\mathrm{R}^{2}$ & $\mathrm{R}^{6}$ & $\mathrm{R}^{12}$ & \\
\hline 40 & $1 R$ & (+)-2-Nor-stephasubin & $\mathrm{H}$ & $\mathrm{CH}_{3}$ & $\mathrm{CH}_{3}$ \\
$\mathbf{4 1}$ & $1 R$ & (+)-Stephasubin & $\mathrm{CH}_{3}$ & $\mathrm{CH}_{3}$ & $\mathrm{CH}_{3}$ \\
$\mathbf{4 2}$ & $1 R$ & (+)-Pycnazanthin & $\mathrm{H}$ & $\mathrm{CH}_{3}$ oder H & $\mathrm{Hoder}_{3}$ \\
\hline
\end{tabular}

Vom Oxyacanthin-Typ sind ebenfalls sehr polare neue BBI-Alkaloide [z.B. das Pangkoramine (23), eine 2-Nor-Verbindung mit 3 phenolischen OH-Gruppen (Abb. 5)] und verschiedene Dehydroderivate (Abb. 6 und 7) beschrieben worden.

Beide neuen Alkaloide vom ThaligosineTyp (Abb. 8) sind ebenso $N$-Oxide wie zahlreiche Beispiele aus der Gruppe der Berbamin-Derivate (Abb. 9). Auch hier wurde eine $\alpha^{\prime}$-Oxo-Verbindung 62 isoliert. Alle neuen BBIAlkaloide aus der Gattung Thalictrum (Abb. 11, 14) stimmen hinsichtlich ihrer Chiralitätszentren mit den für diese Ranunculaceen-Gattung formulierten Regeln $(167,3)$ überein.
Die Tiliacora-Alkaloide mit einem Dibenzodioxin-Kopfteil (Abb. 15) geben sich durch ihre charakteristische blaue Farbreaktion mit salpetersäurehaltiger Schwefelsäure bereits auf der DC-Platte zu erkennen. Bei diesen relativ starren 16-gliedrigen makrocyclischen Diphenylverbindungen wirkt sich die Konfiguration an C-1 und C-1' besonders typisch auf die Verschiebungen $\operatorname{der}^{1}{ }^{1} \mathrm{H}-$ NMR-Signale von $\mathrm{H}-8^{\prime}$ und $\mathrm{H}-10^{\prime}$ aus, so daß bei bekanntem Drehsinn aus den ${ }^{1} \mathrm{H}$-NMR-Spektren unmittelbar auf die absolute Konfiguration geschlossen werden kann (24, $25,27)$. 


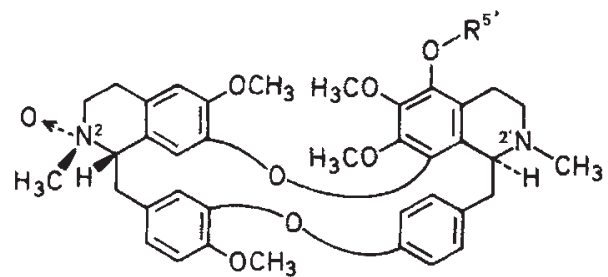

\begin{tabular}{|c|c|c|c|c|}
\hline Verbdg. & abs.Konfg. & Name & $\mathrm{R}^{5}$ & Lit, \\
\hline $\begin{array}{l}43 \\
44\end{array}$ & $\begin{array}{l}1 S, 1^{\prime} S \\
1 S, 1^{\prime} S\end{array}$ & $\begin{array}{l}\text { (-) Thaligosin-2 } \alpha-N \text {-oxid } \\
\text { (-)-Thalrugosaminin-2 } \alpha-N \text {-oxid }\end{array}$ & $\stackrel{\mathrm{H}}{\mathrm{CH}_{3}}$ & $\begin{array}{l}161 \\
161\end{array}$ \\
\hline
\end{tabular}

Abb. 8 Neue BBI-Alkaloide vom Thaligosin-Typ - Typ VIII: $6,7^{*}, 11 \#, 12-5,6,7,8^{*}, 12 \#(1)$.
Die BBI-Alkaloide von Kohatine-Typ (Abb. 16) mit 18 Ringgliedern im Makrocyclus zeigen diese großen Verschiebungsdifferenzen für die analogen ${ }^{1} \mathrm{H}$ NMR-Signale von $\mathrm{H}-8$ und $\mathrm{H}-10$ nicht, es sei denn, daß wie in 88 und 89 beide Ringe $A$ und $B$ aromatisch sind. Einen völlig neuartigen BBI-Typ stellen die Popowia-Alkaloide dar (Abb. 17), wo die beiden Coclaurineinheiten lediglich durch eine Diphenyl-C-C-Bindung miteinander verknüpft sind. Die Strukturaufklärung gelang hier u. a. durch oxidativen Abbau mit $\left(\mathrm{NH}_{4}\right) \mathrm{Ce}\left(\mathrm{NO}_{3}\right)_{6}$.

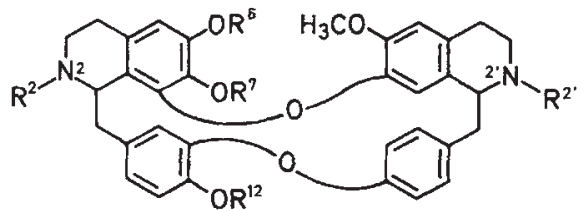

Abb.9 Neue BBI-Alkaloide vom Berbamin-Typ - Typ VIII: $6,7,8^{*}, 11 \#, 12-6,7^{*}, 12 \#(1)$.

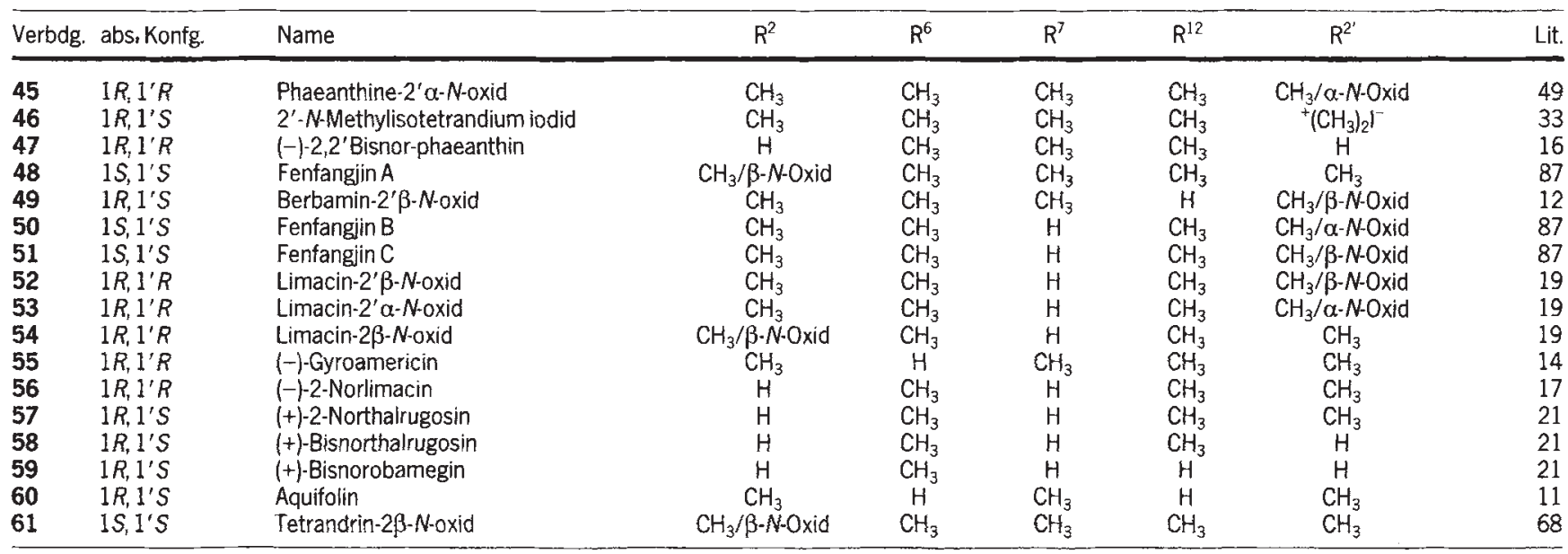<smiles>COC1=CC2=CC=NC3=C(C=C2OCc2cc(OC)c(OC)c(c2)C1Cc1ccc(OC)c(OC)c1)N(C)CCc1ccc(cc1)C3=O</smiles>

Abb. 10 Neue BBI-Alkaloide vom Berbamin-Typ Typ VIII: $6,7,8^{*}, 11 \#, 12-6,7^{*}, 12 \#$ (1).<smiles>COc1ccc2cc1COc1cc3c(cc1OC)CCN(C)[C@H]3Cc1ccc(cc1)CCOc1c(O)c(OC)cc3ccnc(c13)C2</smiles>

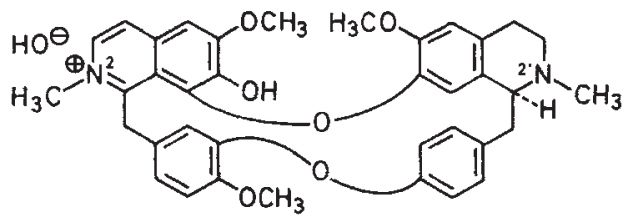

$64 \quad 1^{\prime} S$ Fenfangjin $D$ 


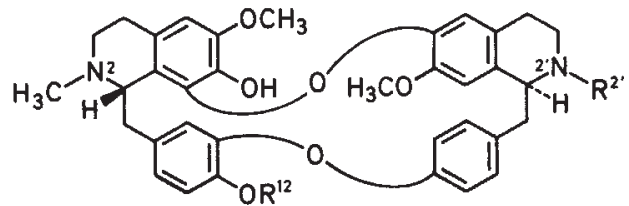

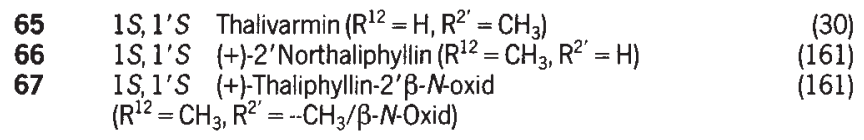

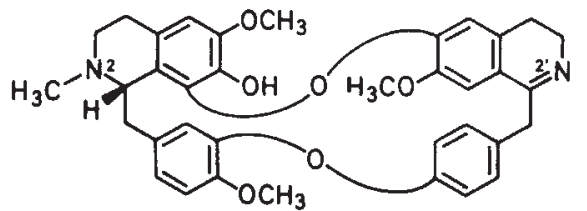

68

1S Thalsivasin

Abb. 11 Neue BBI-Alkaloide vom Thalicberin-Typ Typ IX: 6,7,8*,11\#,12-6* $7,12 \#(1)$.

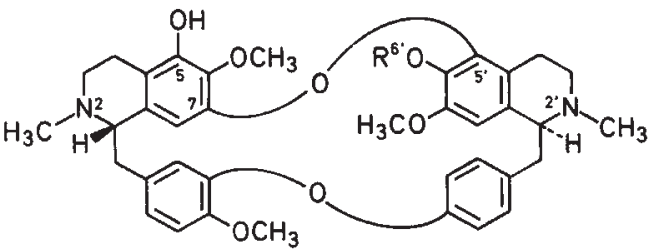

$74 \quad 1 S_{1} 1^{\prime} S(-)-5$-Hydroxythalmin $\left(\mathrm{R}^{6^{\prime}}=H\right)$
75
IS, $1^{\prime} S(-)$-Thalmiculin $\left(\mathrm{R}^{6^{\prime}}=\mathrm{CH}_{3}\right)$

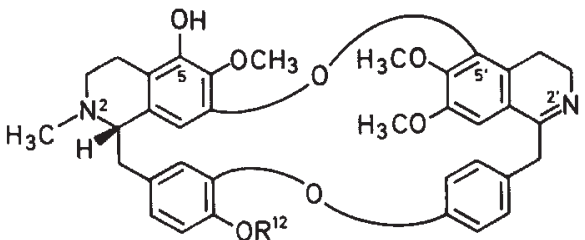

$\begin{array}{llll}76 & \text { is } & (-) \text {-Thaliculimin }\left(\mathrm{R}^{12}=\mathrm{CH}_{3}\right) \\ 77 & \text { is } & \left(-\mathrm{K} \text {-Cultithalimin }\left(\mathrm{R}^{12}=\mathrm{H}\right)\right.\end{array}$

Abb. 14 Neue BBI-Alkaloide vom 5-Hydroxythalmin-Typ - Typ XIVa: $5,6,7^{*}, 11 \#, 12-5^{*}, 6,7,12 \#(1)$.<smiles>[R]c1c2c3c(c(OC)c1OC)OCCc1ccc(cc1)C[C@@H]1c4cc(OC)c(OC)c(c4CCN1C)CCCCCOCc1cc(ccc1OC)C[C@@]3(C)[NH+]([R])CC2</smiles>

Abb. 12 Neue BBI-Alkaloide vom Thalidasin-Typ - Typ Xa: 5,6,7,8*,11\#,12-5*,6,7,12\# (1).

\begin{tabular}{|c|c|c|c|c|c|}
\hline Verbdg. & abs. Konfg. & Name & $\mathrm{R}^{2}$ & $\mathrm{R}^{5}$ & Lit. \\
\hline
\end{tabular}<smiles>COc1cc2c(cc1OC)[C@H](C)N(C)CC2Cc1ccc(O)c(CCOCCc2ccc(CC3=NCCc4c3cc(OC)c(OC)c4CCCCO)cc2)c1</smiles>

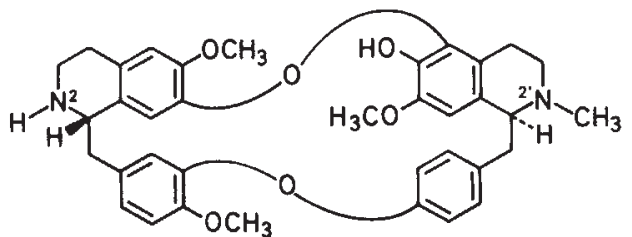

73 $1 S, 1$ 'S (-)-2-Northalmine

Abb. 13 Neue BBI-Alkaloide vom Thalmin-Typ - Typ XIV: $6,7^{*}, 11 \#, 12-5^{*}, 6,12 \#(1)$.
Beide neuen Trilobin-Beispiele (Abb. 19) stellen keine neuartigen BBI-Alkaloide dar. Die Strukturen der neuen Seco-BBI-Alkaloide (Abb. 20) scheinen zu bestätigen, daß es sich um natürliche BBI-Abbauprodukte handelt, allerdings müßte das noch durch Biogenesestudien bewiesen werden.

\section{Ergänzungen zur Struktur bekannter BBI-Alkaloide}

Abb. 21 zeigt einige bekannte BBI-Alkaloide, deren bisherige Strukturen auf Grund neuerer Ergebnisse in die hier wiedergegebenen Formeln berichtigt wurden. So erwies sich Thalrugosinon [224] als 2- $N$-FormylDerivat und nicht als $\alpha, \alpha^{\prime}$-Bisoxo-BBI-Alkaloid (31). Für die beiden Calafantin- $N$-Oxide wurde festgestellt, daß die $N$ Oxid-Funktion sich nicht in Position 2 sondern in 2 '-Stellung befindet (70). Das schon lange bekannte Tiliarin [185] konnte endlich nach erneuter Isolierung als $2^{\prime}$ Nor-Verbindung erkannt werden, die einem 2'-Noryanangcorinin entspricht (vergl. 78; Abb. 15) (37). 
<smiles>COc1cc2c(Oc3cc4c5cc3-c3cc(ccc3OC)C[C@@H]5N(C)CC4)c(c1)C[C@H](c1ccc(O)c(OC)c1)N(C)CC2</smiles>

78

$1 S, 1$ 'S Yanangcorinin

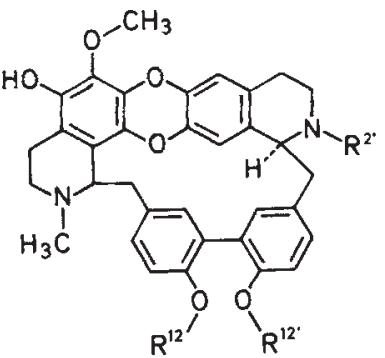

(24)

Abb. 15 Neue BBI-Alkaloide vom Tiliacorin- und Tiliamosin-Typ - Typ XVIII (78): 6,7*,8\#,12-6*7, $\#, 12,(11-11)$ (1) und Typ XIX (79-82): $5,6,7^{*}, 8 \#, 12-6^{*}, 7 \#, 12,(11-11)(1)$.

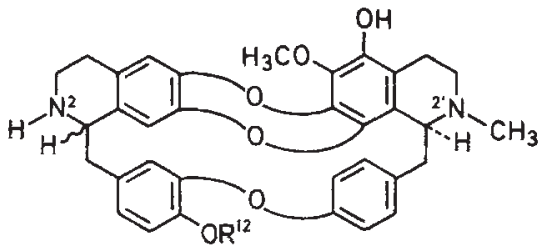

\begin{tabular}{llll}
\hline 83 & $1 R, 1^{\prime} S$ & $(+)-5^{\prime}-$ Hydroxytelobin $\left(\mathrm{R}^{12}=\mathrm{CH}_{3}\right)$ & $(18)$ \\
84 & $1 R_{1} 1^{\prime} S$ & $(+)-5^{\prime}-$ Hydroxyapatelin $\left(\mathrm{R}^{12}=\mathrm{H}\right)$ & $(18)$ \\
85 & $1 S, 1^{\prime} S$ & $(+)-$ Kohatamin $\left(\mathrm{R}^{12}=\mathrm{CH}_{3}\right)$ & $(18)$
\end{tabular}

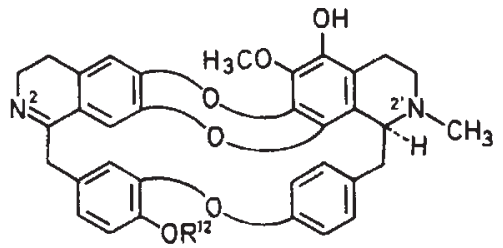

86 1'S (+)-1,2-Dehydrokohatin $\left(R^{12}=H\right)$

87 1's (+)-1,2-Dehydrokohatamin $\left(\mathrm{R}^{12}=\mathrm{CH}_{3}\right)$<smiles>[R20]Oc1ccc(Cc2cc(C[C@@H]3c4cc(OC)c(O[R20])cc4CCN3[R])ccc2[R20])cc1OC</smiles>

Abb. 16 Neue BBI-Alkaloide vom Kohatin-Typ Typ XXIIla: $\left.6^{*}, 7^{* *}, 1\right] \#, 12-5,6,7^{*}, 8^{* *}, 12 \#$.

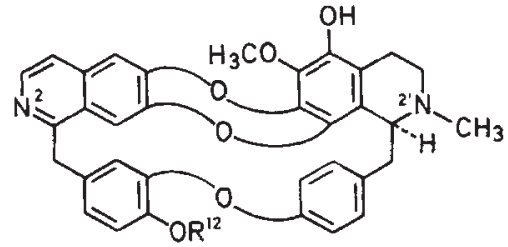

(18)

(18)

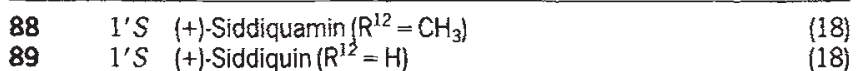

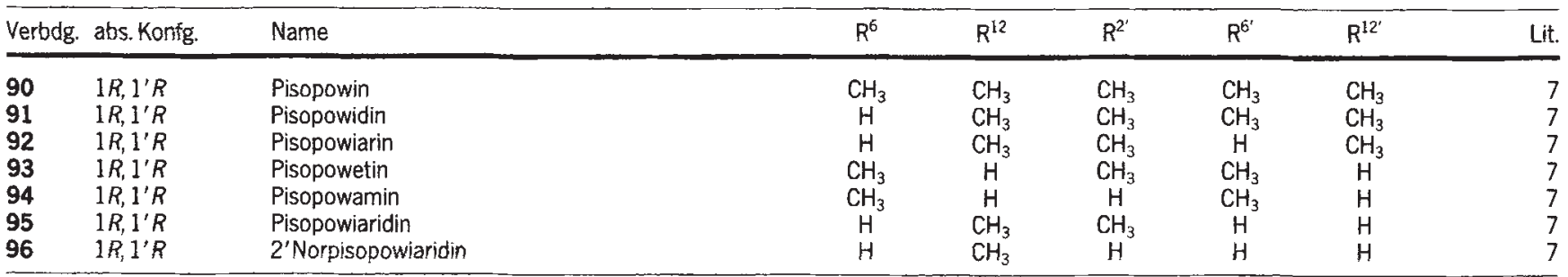


<smiles>[R17]Oc1ccc(C[C@]2(C)c3cc(O)c(OC)cc3CCN2C)cc1OCc1cc2c(cc1OC)CCN(C)[C@H]2Cc1ccc(OC)c(O)c1</smiles>

\section{$97 \quad I S, 1^{\prime} S$ Efatin $\left(\mathrm{R}^{11}=\mathrm{H}, \mathrm{R}^{12}=\mathrm{CH}_{3}\right)$}

$98 \quad 1 S, I^{\prime} S$ Ambrimin $\left(\mathrm{R}^{12}=\mathrm{CH}_{3}, \mathrm{R}^{12}=\mathrm{H}\right)$

(15)

(15)

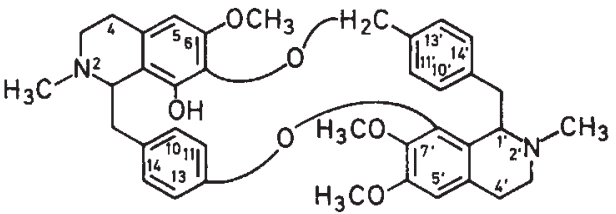

99

(+)-Cycleaneonin

(132)

Abb. 18 Neue Kopf-Schwanz-verknüpfte BBI-Alkaloide - Typ XXVIII (97 und 98): $6,7,10^{*}, 11,12-6,7^{*}, 11,12$ und Typ XXIX (99): $6,7^{*}, 8,12 \#-6,7,8 \#, 15^{*}$.

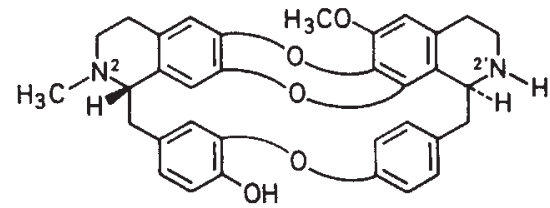

100

1S, 1'S $(+)-2^{\prime}-$ Norcocsulin<smiles>COc1ccc2cc1COCc1cc3c(cc1C2)CCN=C3Cc1ccc(COc2cc3c(cc2OC)CCNC3C)cc1</smiles>

101

1'S (+)-1,2-Dehydro-2'-nortelobin

Abb. 19 Neue BBI-Alkaloide vom Trilobin-Typ Typ XXIII: $6^{*}, 7^{* *}, 11 \#, 12-6,7^{*}, 8^{* *}, 12 \#$.

\section{Synthese und Biosynthese}

Durch Totalsynthese sind in den letzten Jahren nur vereinzelt BBI-Alkaloide hergestellt worden. Der bei den einfachen Benzylisochinolin-Derivaten so bewährte Bishler-Napieralski-Ringschluß führt auch bei den BBI-Alkaloiden zum Ziel, allerdings ist der synthetische Aufwand für die verschiedenen Substituenten meist sehr groß. Nur mit relativ geringer Ausbeute gelingt es, beide Benzylisochinolin-Teile (meist durch Ullmann-Reaktion) miteinander zu verknüpfen, so daß stufenreiche BBI-Totalsynthesen vorläufig nur akademischen Wert besitzen. Bekannt wurden in jüngster Zeit nur Synthesen von Phaeantharin [73] (42), Dauricin [3] (76), Berbamin [57] (76) und von $\mathrm{D}, \mathrm{L}-$ Fangchinolin [58] (41).<smiles></smiles>

$\begin{array}{llll}102 & 1^{\prime} \mathrm{R} & (+) \text {-Auroramin }\left(\mathrm{R}^{6^{\prime}}=\mathrm{CH}_{3}\right) \\ 103 & 1^{\prime} \mathrm{R} & (+) \text {-Maroumin }\left(\mathrm{R}^{6^{\prime}}=\mathrm{H}\right)\end{array}$<smiles>COc1ccc2cc1COc1cc3c(cc1OC)CCN(C)[C@H]3Cc1cc(c(OC)c(OC)c1)C[C@H]2C(=O)O</smiles>

$1041 R \quad(+)-$ Pycmanillin

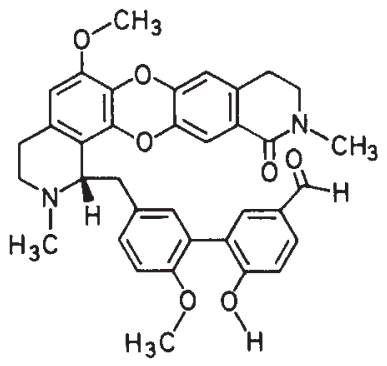

105

is (+)-Secolucidin

Abb. 20 Neue Seco-BBl-Alkaloide vom Seco-oxyacanthin-Typ (102 und 103), Seco-berbamin-Typ (104) und Seco-tiliacorin-Typ (105).

Durch Tracer-Experimente an jungen Pflanzen von Cissampelos pareira wurde gezeigt, daß die stereospezifische Biosynthese von $S$, $R$-Hayatidin [136] durch oxidative Kupplung von $S$ - und $R$ - $N$-Methyl-coclaurin-Einheiten erfolgt, während $R, R$-Isochondodendrin [122] ebenso wie $R, R$-Bebeerin [133] durch oxidative Kupplung zweier $R-N$-Methyl-coclaurin-Einheiten gebildet werden (40). Entsprechend konnte durch Fütterungsexperimente an Blättern von Annona reticulata gezeigt werden, daß $S$-Coclaurin den spezifischen Precursor der meisten Isochinolin-Alkaloide darstellt; - das $R$-Enantiomer wird nicht eingebaut mit Ausnahme von $R, S$-Berbamunine [1], wo $R$-Coclaurin in der $R$-Hälfte des Moleküls erscheint $(160)$.

\section{Ergebnisse pharmakologischer Untersuchungen}

Die neuen Alkaloide sind bisher nur vereinzelt auf eine biologische Wirkung geprüft worden. Die meisten Untersuchungen liegen mit den in ausreichender Menge zugänglichen BBI-Alkaloiden vor.

Das aus Cyclea racemosa isolierte neue Cycleaneonin (99) zeigte bei menschlichen Magenkrebs-Zellkulturen eine deutliche Hemmwirkung (132). Cycleanin [121] besitzt schwache Dopamin-Rezeptor-Inhibitor Eigenschaften (117). Hierdurch inhibiert Cycleanin die Bindung von Spiperone (116). 


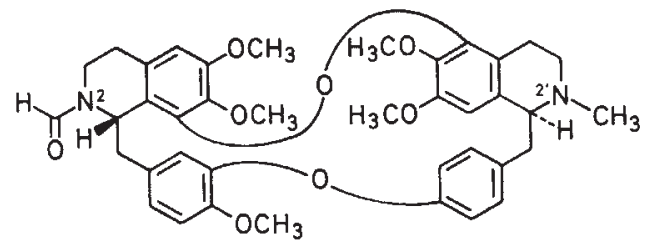

[224] 1S, 1'S Thalrugosinon

(31)

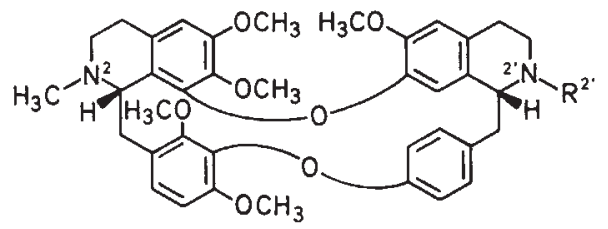

[226] $1 S, 1^{\prime} R$ Calafantin-2' $\alpha-N$-oxid $\left(R^{2}=\mathrm{CH}_{3} / \alpha-N-O x i d\right)$

[227] $1 S$, I'R Calafantin-2' $\beta$ - $N$-oxid $\left(\mathrm{R}^{2^{\prime}}=\mathrm{CH}_{3} / \beta\right.$ - $\mathrm{N}$-Oxid)

(70)

(70)

Für Berbamin [57] wurde immunstimulierende Aktivität bei influenza-infizierten Mäusen beobachtet (80), ferner wurde mehrfach über beachtliche Hemmwirkungen des Berbamins und verschiedener Derivate auf das Calmodulin und calmodulinabhängige Phosphodiesterase berichtet. Als besonders wirksam erwies sich hierbei das partialsynthetische 12-O-(4-Ethoxybutyl)-berbamin $(72,79,82,86)$. Gegen experimentelle myocardiale InfarktBildung bei Ratten und Kaninchen zeigte Berbamin einen schützenden Effect (83) ebenso wie bei experimentellen Arrhythmien (88). Am isolierten Herzmuskel blockiert Berbamin ähnlich wie Verapamil die Calcium-Kanäle $(84,85)$, bei menschlichen KB-Krebszellen soll es teilweise eine Resistenz gegenüber Verbindungen wie Vincristin, Daunomycin und Actinomycin D überwinden (89).

Zahlreiche neuere Untersuchungen liegen für das Cepharanthin [34] vor: Es erhöht den CorticosteronBlutspiegel (92), zeigt einen Leberschutzeffekt (96), verstärkt besser als Berbamin die Aufnahme von Daunomycin in menschliche KB-Zellen (89), und verstärkt die Antitumoraktivität von Vincristin (93). Cepharanthin beeinflußt die Granulocyten-Makrophagen Bildung (99) und ist wirksam gegen experimentelle Mittelohrentzündung (108). Eine Vitamin A bedingte Teratogenität scheint durch Cepharanthin vermindert zu werden (104). Der Einfluß von Cepharanthin und $\alpha$-Tocopherol auf die durch Röntgenstrahlung induzierte Peroxidierung von Lipiden in Lösung wurde untersucht $(97,98)$. Vor Sauerstoffmangel-bedingten Mitochondrienschäden scheint u. a. auch Cepharanthin zu schützen (106). Die verstärkte, inhibierende Wirkung von Cepharanthin auf eine experimentell erzeugte Amyloidosis wurde beschrieben (95). Die Zahl von T-Zellen in den Lymphknoten steigt unter dem Einfluß von Cepharanthin an (90). Es verstärkt die Aufnahme von Adriamycin in Krebszellen (NIH 3T3) und inhibiert den Wiederaustritt (105). Mehrfach wurde auf membranstabilisierende Eigenschaften des Cepharanthin hingewiesen $(91,100,101,103,109)$. Der Hemmeffekt von Cepharanthin auf die Peroxidbildung von polymorphonuclearen Leukozyten und die Abhängigkeit dieser Wirkung verschiedener BBI-Alkaloide von der Zahl ihrer Etherbrücken wurde beschrieben (114). Durch Wechselwirkung des Cepharanthins mit dopaminergen Rezepto-
Abb. 21 Strukturberichtigungen von BBI-Alkaloiden.

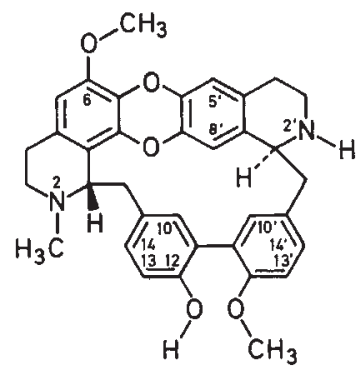

[185] 1S, 1'S Tiliarin

ren wird die Bindung von Spiperone ebenso wie von Berbamin inhibiert (116). Der Einfluß von Cepharanthin auf die Blutgerinnung und Plättchenaggregation (nach vorheriger Arachidonsäure-Stimulation) wurde untersucht $(107,115)$. In mehreren Patenten findet sich die Verwendung von Cepharanthin in Kosmetika (94) und Haarwässern (102, 110, $111,112,113,126)$.

Ein Dauricin-[3]-haltiger Extrakt zeigt ebenso wie Daurinolin [6] aus Menispermum dauricum in vitro antibakterielle Eigenschaften gegenüber zahlreichen pathogenen Stämmen und ist auch wirksam gegen penicillinresistenten Streptococcus pneumoniae (121). Ein Inhibitor-Effekt von Dauricin auf dopaminerge Rezeptoren ist größer als der von Cepharanthin und Cycleanin $(116,117)$. Im Bereich des Herz-Kreislaufsystems zeigt Dauricin am isolierten Rattenherzen eine Verstärkung der coronaren Durchflußrate und eine Minderung des Herzminutenvolumens (120,122). 12-O-Acetyl-dauricin besitzt antiarrhythmische Aktivität, während 12-(4'-Methoxy-phenyl)-dauricin bei Ratten blutdrucksenkend wirkt (125). Durch ADP, Arachidonsäure oder Collagen induzierte Plättchenaggregation bei Kaninchen wird durch Dauricin verhindert (118). Bei experimentellen Oedemen an der Maus wirkt Dauricin antiphlogistisch (119).

Guattegaumerin [234] erwies sich in Vorversuchen als cytotoxisch und antimitotisch wirksam bei verschiedenen Krebszellkulturen in Dosen von $10 \mu \mathrm{g} / \mathrm{ml}$, wobei B16-Melanomzellen und L 1210-Zellkulturen am stärksten beeinflußt werden (45). Der Rohextrakt von Guatteria gaumeri, der als Hauptbestandteile $\alpha$-Asaron und Guattegaumerin enthält, wirkt cholesterinspiegelsenkend (56). Fenfangjin D (64) aus Stephania tetrandra zeigt in vitro ACE-hemmende Eigenschaften (39); ebenso wie die altbekannten BBIAlkaloide Tetrandrin [76], Fangchinolin [61] und Tetrandrin$N$-oxide [78] $(68,87,128)$. Die durch reduktive Etherspaltung aus Tetrandrin erhaltenen Benzylisochinolinderivate $O$-Methyl-armepavin und $N$-Methyl-coclaurin zeigen diese Wirkung auf ACE nur in höheren Dosen (163). 
Tabelle 1 Botanische Herkunft der neuen BBI-Alkaloide.

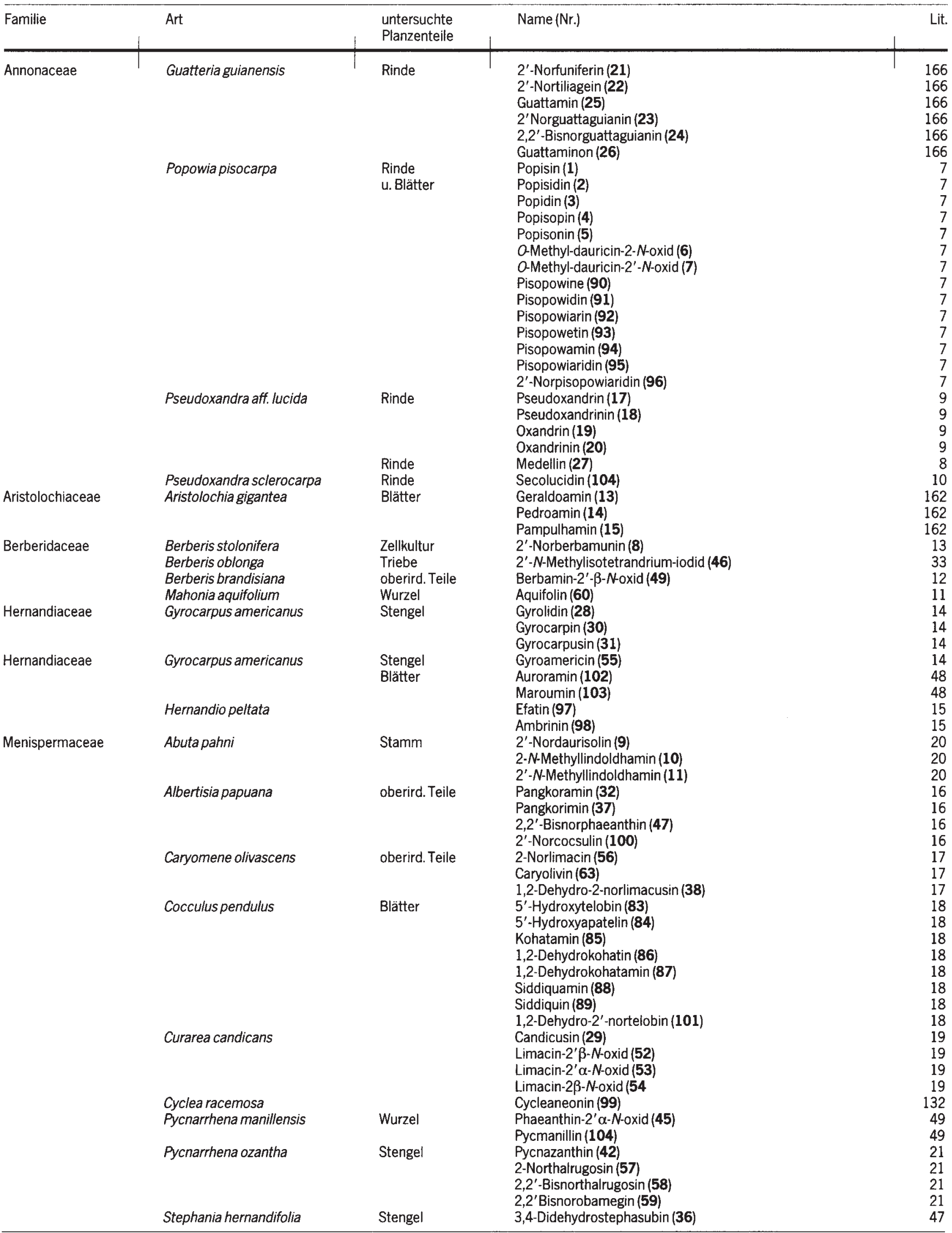


Tabelle 1 (Fortsetzung).

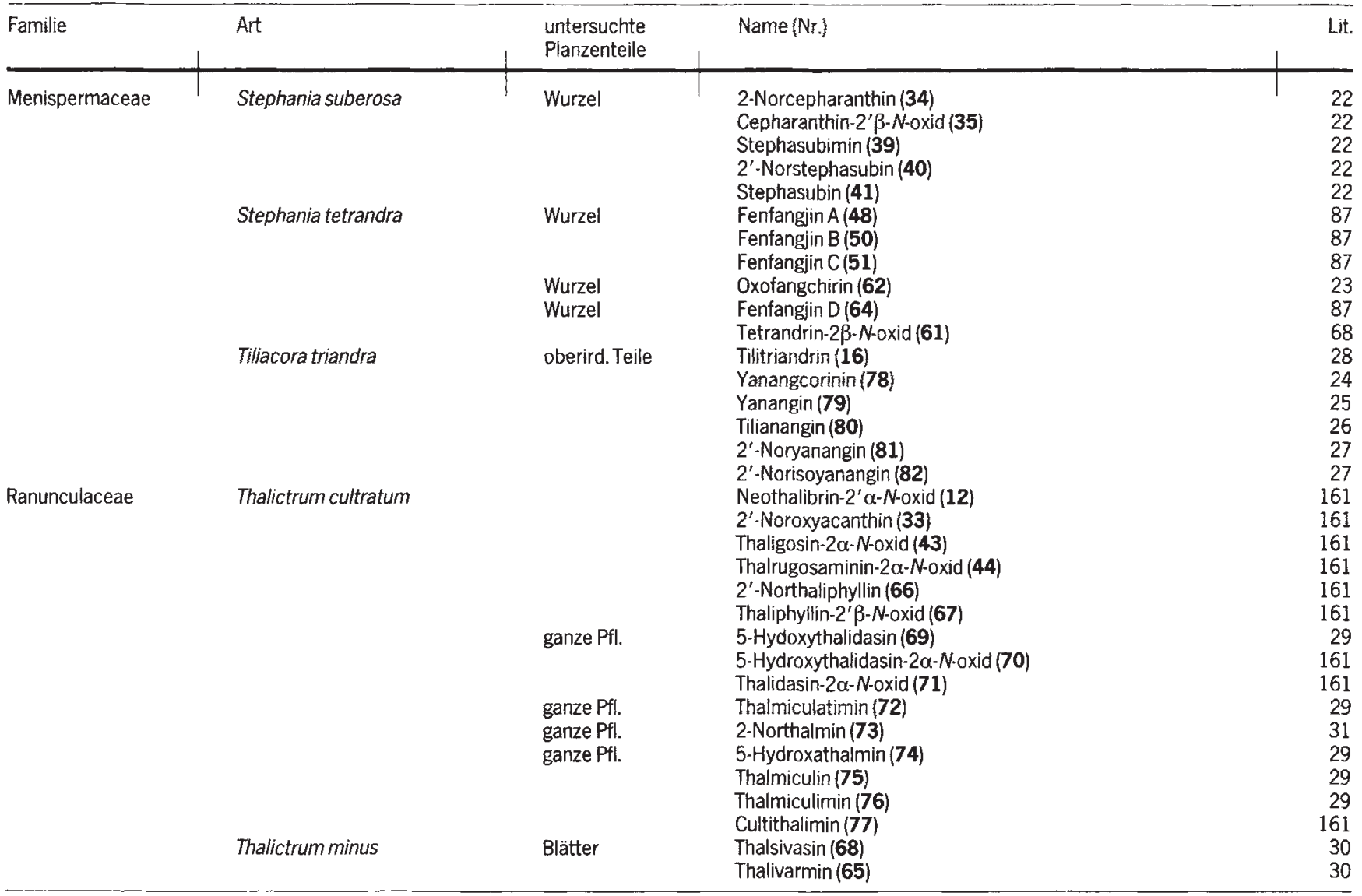

Das muskelrelaxierende Tetrandrindimethoiodid zeigte ebenfalls starke Blutdruckeffekte durch Beeinflussung des arteriellen und pulmonalen Gefäßwiderstands an Pentobarbital-behandelten Hunden $(44,150)$. Auch Tetrandrin [76] scheint als potenter Vasodilatator die Durchblutung arterieller Gefäße ohne eine signifikanten negativ inotropen Effekt zu verbessern $(55,129,148)$. Eine Reihe von Untersuchungen befassen sich mit dem hemmenden Einfluß von Tetrandrin auf das Calmodulin System $(149,141)$ und seine Calcium-antagonistische Wirkung $(123,124,127,131,138,143,144,147)$. Tetrandrin wird ferner wegen seiner Wirkung auf die Mikrotubuli und auf die Monozyten für die Behandlung von Silicosis diskutiert $(134,136,137,140)$. Schon lange bekannt ist die cytostatische Wirkung von Tetrandrin, neuere Untersuchungen befassen sich mit dem Einfluß des Tetrandrins auf die DNASynthese (130) und seine immunsuppressiven Eigenschaften (133). Tetrandrin inhibiert die Histamin-Freisetzung aus den Mastzellen $(135,146)$ und wirkt als Sauerstoff-Radikalfänger antioxidierend und antiphagocytotisch, womit auch die antiphlogistischen Effekte erklärt werden (134). Nach i.m. Applikation erwies sich Tetrandrin ähnlich antiphlogistisch wirksam wie Phenylbutazon (81). An isolierten Kaninchen-Eileitern inhibiert Tetrandrin spontane und durch Phenylephrin ausgelöste Kontraktionen und verzögert so den Eitransport (145). Angaben zu Biotransformation von Tetrandrin und anderen cytostatisch wirksamen Alkaloiden finden sich in einer neueren Übersichtsarbeit (142).
Trilobin [163] und Isotrilobin [157] erwiesen sich ebenso wie Tetrandrin als potente, dem Phenylbutazon vergleichbare Antiphlogistika $(81,154)$. Dimethyltrilobin-iodid zeigt erwartungsgemäß muskelrelaxierende Effekte $(152,154,155,156)$. Für die Tiliacora-Alkaloide Tiliacorin [118], Tiliacorinin [119], Nortiliacorinin A [116] und 2 weitere, nicht identifizierte Alkaloide aus Tiliacora triandra wurde kürzlich über eine in-vitro Antimalaria-Aktivität berichtet (46).

\section{Ergebnisse mit Zellkulturen}

In jüngster Zeit wurde über Zellkulturen der Gattungen Berberis und Stephania berichtet. An Callus Zellkulturen von zahlreichen Berberis-Arten wurde die Biogenese der verschiedenen Berbamin- und Aporphin-Alkaloide untersucht (13). Die Identifizierung der bekannten Verbindungen gelang über Massen-, NMR- und CD-Spektren. Von Berberis stolonifera wurde dabei das bisher unbekannte 2-Norberbamunin (8) isoliert. In einer weiteren Arbeit der gleichen Arbeitsgruppe wird der Einbau der Biogenesevorstufen $S$ - und $R$-Nor-coclaurinderivate in die verschiedenen BBI-Alkaloide und Aporphin-Alkaloide untersucht (50). 
Tabelle 2 Neue botanische Herkunft bekannter BBI-Alkaloide.

\begin{tabular}{|c|c|c|c|c|}
\hline Familie & Art & $\begin{array}{l}\text { untersuchte } \\
\text { Planzenteile }\end{array}$ & isolierte Alkaloide (Nr.) & Lit \\
\hline \multirow[t]{2}{*}{ Annonaceae } & $\begin{array}{l}\text { Guatteria guianensis } \\
\text { Pseudoxandra sclerocarpa }\end{array}$ & $\begin{array}{l}\text { Rinde } \\
\text { Rinde }\end{array}$ & $\begin{array}{l}\text { Tilliagein [27] } \\
\text { Berbamunin [1] } \\
\text { Thaligrisin [252] } \\
\text { Homoaromolin [42] }\end{array}$ & $\begin{array}{r}166 \\
10 \\
10 \\
10\end{array}$ \\
\hline & Popowia pisocarpa & Rinde & $\begin{array}{l}\text { Dauricin [3] } \\
\text { O-Methyldauricin [12a] } \\
\text { Dauricolin [5] }\end{array}$ & \\
\hline \multirow[t]{11}{*}{ Berberidaceae } & Berberis brandisiana & oberird. Teile & $\begin{array}{l}\text { Isotetrandrin [62] } \\
\text { Berbamin [57] } \\
\text { Pendulin [72] }\end{array}$ & $\begin{array}{l}12 \\
12 \\
12\end{array}$ \\
\hline & Berberis cretica & ganze Pfl. & $\begin{array}{l}\text { Oxyacanthin [48] } \\
\text { Obaberin [46] } \\
\text { Aromolin [31] } \\
\text { Berbamin [57] } \\
\text { Isofangchinolin [79] } \\
=\text { Thalrugosin } \\
=\text { Thaligin } \\
\text { Isotetrandrin [62] }\end{array}$ & $\begin{array}{l}69 \\
69 \\
69 \\
69 \\
69 \\
69\end{array}$ \\
\hline & Berberis koreana & & $\begin{array}{l}\text { Berbamin [57] } \\
\text { Oxyacanthin [48] } \\
\text { Obamegin [71] } \\
\text { Aromolin [31] }\end{array}$ & $\begin{array}{l}67 \\
67 \\
67 \\
67\end{array}$ \\
\hline & Berberis pseudambalata & & $\begin{array}{l}\text { OMethyloxycanthin [46] } \\
\text { Oxyacanthin [48] }\end{array}$ & $\begin{array}{l}59 \\
59\end{array}$ \\
\hline & Berberis regeliana & & Berbamin [57] & 62 \\
\hline & Berberis wilsoniae & & $\begin{array}{l}\text { Berbamin [57] } \\
\text { Isotetrandrin [62] }\end{array}$ & $\begin{array}{l}57 \\
57\end{array}$ \\
\hline & Berberis oblonga & & $\begin{array}{l}\text { Berbamin [57] } \\
\text { Oxyacanthin [48] }\end{array}$ & $\begin{array}{l}33 \\
33\end{array}$ \\
\hline & Mahonia aqufolium & Wurzel & Baluchistin [188] & 11 \\
\hline & & Wurzel & $\begin{array}{l}\text { Aromolin [32] } \\
\text { Obamegin [71] }\end{array}$ & $\begin{array}{l}34 \\
34\end{array}$ \\
\hline & & & Berbamin [57] & 64,65 \\
\hline & & & $\begin{array}{l}\text { Oxyacanthin [48] } \\
\text { Isotetrandrin [62] }\end{array}$ & $\begin{array}{l}64 \\
64\end{array}$ \\
\hline \multirow[t]{5}{*}{ Hernandiaceae } & Gyrocarpus americanus & & Grisabin $[10]$ & \\
\hline & & & Limacin [64] & \\
\hline & & & Phaeanthin [64] & \\
\hline & & & Isotetrandrin [62] & \\
\hline & & & 7'-0-Methyllimacusin & 14 \\
\hline \multirow{23}{*}{ Menispermaceae } & Albertisia papuana & Triebe & Bisnoraromolin [32] & $\begin{array}{l}16 \\
16\end{array}$ \\
\hline & & & $\begin{array}{l}\text { Cocsulin [153] } \\
\text { Cocsolin [152] }\end{array}$ & $\begin{array}{l}16 \\
16\end{array}$ \\
\hline & & & O-Methylcocsolin [239] & 16 \\
\hline & & & Lindoldhamin [11] & 16 \\
\hline & & & Daphnolin [38] & 10 \\
\hline & & & Daphnandrin [37] & $\begin{array}{l}16 \\
16\end{array}$ \\
\hline & & & Apatelin [187] & $\begin{array}{l}16 \\
66\end{array}$ \\
\hline & Albertisia laurifolia & Wurzel & Aromolin [31] & $\begin{array}{l}66 \\
66\end{array}$ \\
\hline & & & $\begin{array}{l}\text { Daphnolin [38] } \\
\text { Cocsolin [152] }\end{array}$ & $\begin{array}{l}b 6 \\
66\end{array}$ \\
\hline & & & Apatelin [187] & 66 \\
\hline & & & N-Methylapatelin [207] & 66 \\
\hline & Cocculus hirsutus & Blätter & $\begin{array}{l}\text { Trilobin [163] } \\
\text { Isotrilobin [157] }\end{array}$ & $\begin{array}{l}58 \\
58\end{array}$ \\
\hline & Cyclea hypoglauca & & Insulanolin [169] & 7 \\
\hline & & & Insularin [170] & \\
\hline & Pachygone loyaltiensis & & Daphnandrin [37] & 3 \\
\hline & & & Daphnolin [38] & \\
\hline & & & $N, N^{\prime}$-Bisnoraromolin [32] & $\begin{array}{l}3 \\
3\end{array}$ \\
\hline & & & Isotrilobin [157] & $\begin{array}{l}5 \\
5\end{array}$ \\
\hline & & & $\begin{array}{l}\text { O-Methylcocsolin [239] } \\
\text { Apatelin [187] }\end{array}$ & $\begin{array}{l}35 \\
35\end{array}$ \\
\hline & & & 1,2-Dehydroapatelin [193] & \\
\hline & & & 1,2-Dehydrotelobin [194] & \\
\hline & Pycnarrhena ozantha & Stengel & 2-Norobamegin [69] & 1 \\
\hline & & & $\begin{array}{l}\text { 2-Norberbamin [68] } \\
\text { Daphnolin [38] }\end{array}$ & $\begin{array}{l}21 \\
21\end{array}$ \\
\hline
\end{tabular}


Tabelle 2 (Fortsetzung).

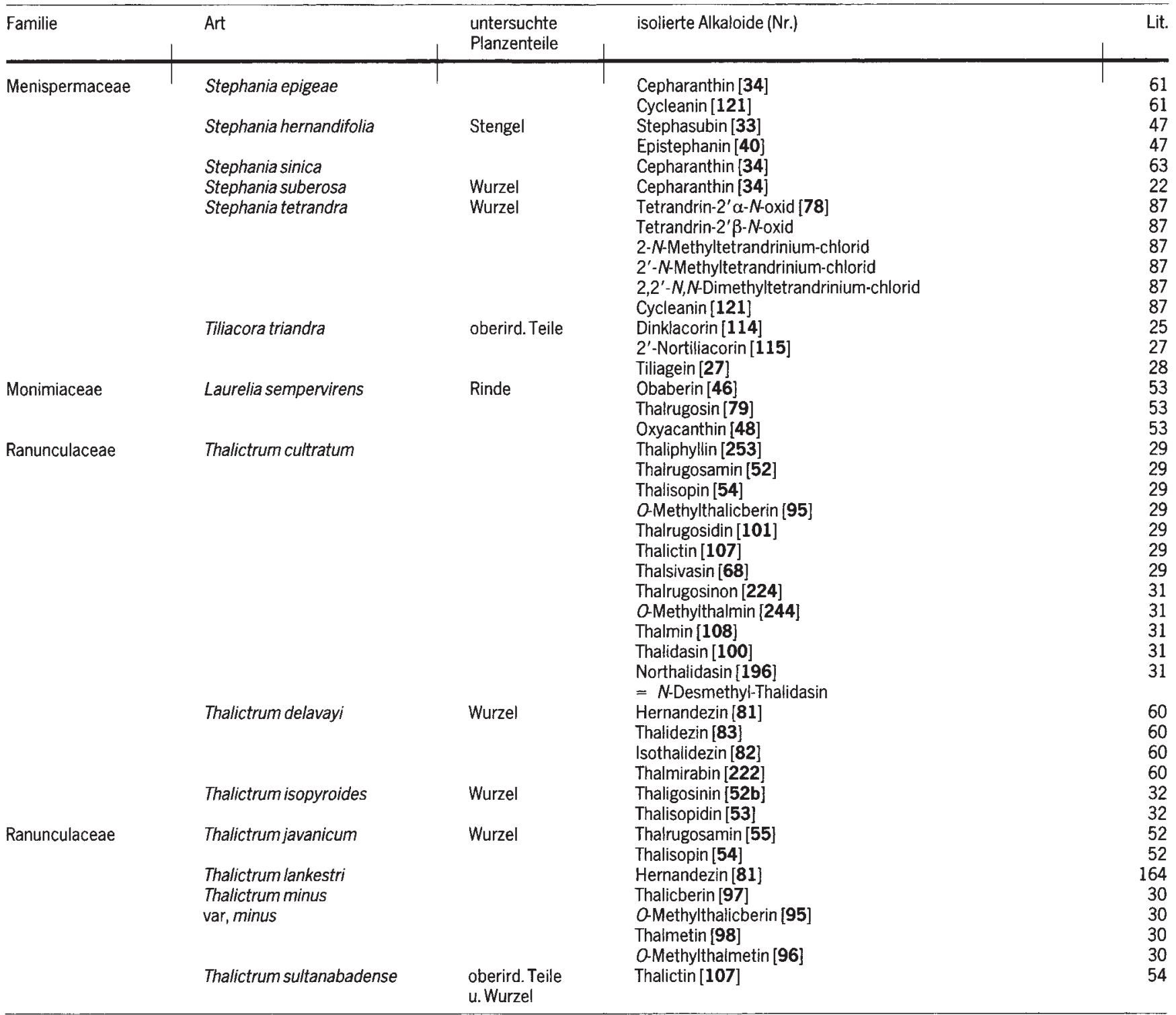

* Erstmals als Naturprodukt isoliert.

In Zellkulturen der Wurzel von Stephania cepharantha wurden mindestens 5 verschiedene BBI-Alkaloide gefunden, - das Fehlen von Cepharanthin und Cepharanolin wird mit der extrem niedrigen Aktivität der Methylendioxy-gruppe bildenden Enzyme begründet (38). Der Gehalt an BBI-Alkaloiden ist abhängig von den Kulturbedingungen der Wurzel und wesentlich höher als in den Wurzeln der ganzen Pflanze (51).

\section{Danksagung}

Frau Apothekerin E. Büse danke ich für die umfangreichen und sorgfältigen Literaturrecherchen.

\section{Literatur}

Guha, K. P., Mukherjee, B. (1979) J. Nat. Prod. 42, 1.

2 Schiff, P. L. Jr. (1983) J. Nat. Prod. 46, 1.

3 Schiff, P. L. Jr. (1987) J. Nat. Prod. 50, 529.

4 Shamma, M., Moniot, J. (1976) Heterocycles 4, 1817.

5 Buck, K. T. (1987) Alkaloids, Academic Press, New York, Vol. 30, p. 1.

6 Baser, K. H. (1986) Stud. Org. Chem. (Amsterdam) 26; New Trends Nat. Prod. Chem. (1986), 45.

7 Jossang, A., Leboeuf, M., Cave, A., Sevenet, T. (1986) J. Nat. Prod. $49,1018$.

8 Cortes, D., Saez, D., Hocquemiller, R., Cave, A. (1986) Heterocycles 24,607

9 Cortes, D., Hocquemiller, R., Cave, A., Saez, J., Cave, A. (1986) Can. J. Chem. 64,1390

10 Cortes, D., Hocquemiller, R., Cave, A., Saez, J. (1986) J. Nat. Prod. 49,854 .

11 Kostalova, D., Uhrin, D., Hrochova, V., Tomko, J. (1987) Collect Czech. Chem. Commun. 52, 242.

12 Hussain, S. F., Siddiqui, M. T., Khan, L., Freyer, A. J., Guinaudeau, H., Shamma, M. (1986) J. Nat. Prod. 49, 538. 
${ }^{13}$ Cassels, B., Breitmaier, E., Zenk, M. (1987) Phytochemistry 26, 1005.

14 Chalandre, M. C., Bruneton, J., Cabalion, P., Guinaudeau, H. (1986) J. Nat. Prod. 49, 101.

15 Chalandre, M. C., Guinaudeau, H., Bruneton, J. (1985) C. R. Acad. Sci. Ser. 2301,1185

16 Lavault, M., Bruneton, J., Cave, A., Chan, K. C., Deverre, R. (1987) Can. J. Chem. 65, 343.

17 Lavault, M., Fournet, A., Guinaudeau, H., Bruneton, J. (1986) Chem. Pharm. Bull. 34, 1148.

18 Guinaudeau, H., Bashir, M., Colton, M. D., Freyer, A. J., Shamma, M., Jehan, K., Nilofar, A., Attar-ur-Rahman (1987) Phytochemistry 26,829 .

19 Lavoult, M., Fournet, A., Guinaudeau, H., Bruneton, J. (1985) J. Chem. Res. (M) 2786.

20 Dute, P., Weber, J. F., Fournet, A., Cave, A., Bruneton, J. (1987) Phytochemistry 26, 2136.

21 Abouchacra, M. L., Leboeuf, M., Guinaudeau, H., Cave, A. (1987) J. Nat. Prod. 50, 375.

22 Patra, A., Freyer, A. J., Guinaudeau, H., Shamma, M., Tantisewie, B., Pharadai, K. (1986) J. Nat. Prod. 49, 424.

${ }^{23} \mathrm{Hu}$, T. M., Zhao, S.-X. (1986) Yaoxue Xuebao 21, 29; C. A. 104, 221975q

${ }^{24}$ Pachaly, P., Tan, T. J., Khosravian, H., Klein, M. (1986) Arch. Pharm. (Weinheim) 319), 126.

25 Pachaly, P., Tan, T. J. (1986) Arch. Pharm. (Weinheim) 319, 841.

26 Pachaly, P., Tan, T. J. (1986) Arch. Pharm. (Weinheim 319, 872.

27 Pachaly, P., Khosravian, H. (1988) Planta Med. 54, 433.

28 Pachaly, P., Khosravian, H. (1988) Planta Med. 54, 516.

${ }^{29}$ Hussain, S. F., Freyer, A. J., Guinaudeau, H., Shamma, M. (1986) J. Nat. Prod. $49,488$.

30 Baser, K. H. C., Kirimer, N. (1985) Planta Med. 448.

31 Hussain, S. F., Guinaudeau, H., Freyer, A., Shamma, M. (1985) J. Nat. Prod. 48, 962.

32 Al-Khalil, S., Schiff, P. L. Jr. (1986) Phytochemistry 25, 935

33 Karimov, A., Lutfullin, K. L. (1986) Khim. Prir. Soedin. 249; C. A. $105,197034 \mathrm{e}$.

34 Kostalova, D., Hrochova, V., Tomko, J. (1986) Chem. Pap. 40, 389.

${ }^{35}$ Leboeuf, M., Abouchacra, M. L., Cave, A., Debray, M. (1987) Plant. Med. Phytother. 21, 106.

${ }^{36}$ Da Rocha, A. I., Luz, A. I. R., Da Silva, M. F. (1984) Acta Amazonica $14,244$.

${ }^{37}$ Guinaudeau, H., Freyer, A. J., Shamma, M., Mitra, S. K., Roy, A. K., Mukherjee, B. (1985) J. Nat. Prod. 1985, 651.

${ }^{38}$ Sugimoto, Y., Sugimura, Y., Yamada, Y. (1988) Phytochemistry 27, 1379.

${ }^{39}$ Ogino, T., Sato, S., Sasaki, H., Chin, M., (1987) Jpn. Kokai Tokkyo Koho JP 62, 294, 684 [87, 294, 684]; C. A. 109, 55030].

40 Bhakuni, D. S., Jain. S., Chaturvedi, R. (1987) Tetrahedron 43, 3975.

41 Deng, D., Pang, B. (1986) Huaxue Xuebao 44, 39; C. A. 105 , $172803 \mathrm{~s}$

42 Knabe, J., Hanke, B. (1986) Arch. Pharm. (Weinheim) 319, 950.

43 Bick, I. R. C., Brenner, J. B., Le Van Thuc, Wiriyachitra, P. (1986) J. Nat. Prod. 49,373

44 Ying, S. (1987) Chin. Med. J. 100, 293; C. A. 107, 228940v.

45 Leclercq, J., Quetin, J., De Pauw-Gillet, M. Cl., Bassleer, R., Angenot, L. (1987) Planta Med. 116.

46 Dechatiwongse, T., Chavalittumrong, P., Nutakul, W. (1987), Bull. Dept. Med. Sci. (Bangkok) 29, 33.

47 Patra, A., Mandal, T. K., Mukhopadhyay, P. K., Ranu, B. C. (1988) Phytochemistry 27, 653 .

48 Dute, P., Chalandre, M. C., Cabalion, P., Bruneton, J. (1988) Phytochemistry 27,655

49 Regalado, J. C. Jr., Gao, C., Fu, E., Lin, F., Lin, M., Wong, L. K., Schiff, P. L. Jr. (1987) Heterocycles 26, 2573.

50 Stadler, R., Loeffler, S., Cassels, B. K., Zenk, M. H. (1988) Phytochemistry 27, 2557.

51 Sugimoto, Y., Sugimura, Y., Yamada, Y. (1988) Agric Biol. Chem. 52,1495

52 Sahai, M., Sinha, S. C., Ray, A. B. (1985) J. Nat. Prod.48, 669

${ }^{53}$ Cassels, B. K., Urzura, A. (1985) J. Nat. Prod. 48, 671.

54 Baser, K. H. C., Ögütveren, M., Bisset, N. (1985) J. Nat. Prod. 48 , 672
55 Kubota, K., Ogino, T., Sasaki, H., Chin, M. (1987) Jpn. Kokai Tokkyo Koho JP 62, 209, 018 [87, 209, 018]; C. A. 108, 173549n (1988).

56 Leclercq, J., Dehaussy, H., Goblet, M. C., Wauters, J. N., Angenot, L. (1985), J. Pharm. Belg. 40, 251.

57 Hrochova, V., Kostalova, D. (1985) Chech. Farm. 34, 412; C. A. 104, $106264 \mathrm{v}$.

58 Ahmad, V. U., Rashid, T. (1986) J. Chem. Soc. Pak. 8, 537; C. A. 106, 99448u.

59 Pant, N., Garg, H. S., Bhakuni, D. S. (1986) Fitoterapie 57, 427; C. A. $107,172419 n$.

60 Lin, L., Zhang, J, Xu, C., Chen, Z. (1987) Zhongcaoyao 18, 2; C. A. $107,93538 \mathrm{x}$.

61 Chen, Z., Yang, S., Ding, X. (1985) Nangjing Yixueyuan Xuebao 5 , 203; C. A. 104, 106271v.

62 Yusufbekov, Kh., Yu, K. S., Sadykov, Y. D., Aknazarov, O. A., Poryadina, T. V. (1985) Dokl. Akad. Nauk. Tadzh. SSR 28, 712; C. A. 105 , $3564 \mathrm{j}$.

63 Zhi-Da, M., Ge, L., Guan-xi, X., Inuma, M., Tanaka, T., Mizuno, M. (1985) Phytochemistry 24, 3084.

${ }^{64}$ Khusainova, Kh. Sh., Sadykov, Y. D. (1986) Izv. Akad. Nauk. Tadzh. SSR, OtD. Fiz.-Mat. Khim. Geol. Nauk. 41; C. A. 105, 130695c.

65 Slavik, J., Bochorakova, J., Kostalova, D., Hrochova, V. (1985) Chem. Pap. 39, 537

${ }^{66}$ Xue, Z., Wu, Y., Zhang, P., Ma, J., He, J. (1985) Zhiwu Xuebao 27. $630 ;$ C. A. $104,106299 \mathrm{k}$.

${ }^{67}$ Hrochova, V., Kostalova, D. (1987) Cesk. Farm. 36, 457; C. A. 108 , $128549 \mathrm{~b}$.

68 Ogino, T., Sato, S., Sasaki, H., Chin, M. (1988) Jpn. Kokai Tokkyo Koho JP 62, 205, 084; C. A. 109, 79696g.

${ }^{69}$ Ross, S., Gozler, T., Freyer, A. J., Shamma, M., Cubukcu, B. (1986) J. Nat. Prod. 49, 159

70 Leet, J. E., Freyer, J. A., Minard, R. D., Shamma, M. (1985) J. Chem. Soc., Perkin Trans. 1, 1565.

71 Fang, X., Qiang, L., Shen, P., Shi, Z. (1985) Zhongcaoyao 16, 536; C. A. $104,145508 \mathrm{x}$.

72 Zhang, S., Xu, Y. (1988) Shengwu Huaxu Yu Shengwu Wuli Xuebao 20,13; C. A. $109,124847 \mathrm{v}$.

73 Zhou, H., Wang, H., Wang, C. (1986) Yaoxue Tongbao 21, 648; C. A. $108,226930 p$.

74 Wang, C. Y., Zhang, D. H., Guo, Y. L., Zhong, H. M., Wen, M. L. (1987) Anal. Chim. Acta 196, 299.

75 Ye, X., Li, X. (1987) Yaowu Fenxi Zazhi 7, 77; C. A. 107, 32489k.

76 Huang, W., Yang, Z., Peng, S. (1987) Yiyao Gongye 18, 447; C. A. $109,6776 y$.

77 Dou, S., Guan, Z., Shan, G., Zhou, Y., Xie, R. (1987) Jiegou Huanxue 6, 84 ; C. A. $108,150785 x$.

${ }^{78}$ Kuz'mitskaya, A. E. (1986) Farm. Zh. (Kiev) 5, 44; C. A. 106, 9438p.

79 Xu, Y., Liu, J., Zhang, S., Liu, L. (1987) Biochem. J. 248, 985.

80 Jin, L., Sui, W. (1986) Zhongguo Yaoli Xuebao 7, 475; C. A. 105, $202881 \mathrm{k}$.

81 Yamahara, J. (1986) Gendai Toyo Igaku 7, 49; C. A. 106, 131115w.

${ }^{2}$ Hu, Z., Chen, S., Hao, Z., Huang, W., Peng, S. (1988) Zhongguo Yaoke Daxue Xuebao 19, 78; C. A. 109, 66356w.

83 Wang, X., Yang, B., Li, Y., Li, W.(1986) Zhongguo Yaoli Xuebao 7, 231; C. A. 105, $18097 \mathrm{~h}$

${ }^{84} \mathrm{Li}, \mathrm{F} ., \mathrm{Bao}, \mathrm{L} ., \mathrm{Li}, \mathrm{W} .(1985)$ Yaoxue Xuebao 20, 859; C. A. 104 , 199836w.

${ }^{85}$ Li, N., Li, W., Li, Y. (1986) Zhongguo Yaoli Xuebao 7, 222; C. A. 105 , $18095 f$.

86 Xu, Y., Zhang, S. (1986) Biochem. Biophys. Res. Commun. 140, 461; C. A. 106,27670 n

87 Ogino, T., Sato, T., Sasaki, H., Chin, M., Mitsuhashi, H. (1988) Heterocycles $27,1149$.

88 Yang, B., Wang, X., Li, Y., Li, W. (1987) Yaoxue Xuebao 22, 700; C. A. $107,228843 \mathrm{r}$.

89 Shirashi, N., Akiyama, S., Nakagawa, M., Kobayashi, M., Kuwano, M. (1987) Cancer Res. 47, 2413; C. A. 107, 32746s.

90 Nihashi, Y., Koga, Y., Gondo, H., Taniguchi, K., Nomoto, K. (1985) Immunobiology (Stuttgart) 170, 351.

91 Matsuno, T., Orida, K., Sato, E., Inoue, T., Utsumi, K. (1986) Igaku to Xakugaku 16, 445; C. A. $107,126480 \mathrm{c}$.

92 Yoshikawa, N., Seyama, Y., Yamashita, S., Akasu, M., Inoue, H. (1986) Nippon Yakurigaku Zasshi 87, 99; C. A. 104, 141780g.

93 Kato, T., Suzumura, Y. (1987) J. Nat. Cancer Inst. 79, 527; C. A. 107, $228584 \mathrm{~g}$ 
94 Akasu, M. (1985) Jpn. Kokai Tokkyo Koho JP 60, 209, 508; C. A. $104,74820 \mathrm{e}$

95 Nagasawa, T., Ishihara, T., Uchino, F. (1985) Yamaguchi Igaku 34, 369 ; C. A. 104, 102034x.

96 Takehara, Y., Yamasaki, M., Fujii, Y., Yoshioka, T. (1985) Geka to Taisha, Eiyo 19, 323; C. A. 104, 123125 t.

${ }^{97}$ Shiraishi, N., Joja, I., Kuroda, M., Fujishima, M., Miyake, M., Aono, K. (1985) Physiol. Chem. Phys. Med. NMR 17, 243; C. A. 104, $144669 p$

98 Joja, I. (1985) Okayama Igakkai Zasshi 97, 235; C. A. 104 , $144760 \mathrm{~m}$.

99 Uno, N., Matsuoka, N., Uchida, T., Shimizu, N., Katayama, N., Minami, N., Shirakawa, S. (1985) Igaku no Ayumi 135, 595; C. A. 104, $144766 t$.

${ }^{100}$ Kometani, M., Sato, T., Fujii, T. (1986) Thromb. Res. 42, 567; C. A. $105,4193 z$.

101 Sato, E., Takehara, Y., Sasaki, J., Matsuno, T., Utsumi, K. (1986) Cell. Struct. Funct. 11, 125; C. A. 105, 59361h.

102 Okazaki, T., Suetsugu, M., Tsuji, Y., Kino, A., Yoshida, T. (1987) Jpn. Kokai Tokkyo Koho JP 62, 84, 014; C. A. 107, 46052z.

103 Miyahara, M., Takahashi, M., Kawashima, H., Okimasu, E., Nobori, K., Yamamoto, H., Kobayashi, S., Utsumi, K. (1986) Curr. Clin. Pract. Ser. 36, 168; C. A. 105, 188820 f.

104 Matsuda, Y., Ueda, S., Sanei, T., Ogura, Y., Nagai, H., Susuki, K. (1985) Ear. Res. Jpn. 16, 273; C. A. 106, 4084k.

105 Nagaoka, S., Kawasaki, S., Karino, Y., Sasaki, K., Nakanishi, T. (1987) Eur. J. Cancer. Clin. Oncol. 23, 1297; C. A. 107, 228604p.

106 Miyahara, M., Takahashi, M., Kawajima, H., Okimasu, H., Nobori, K., Yamamoto, H., Kobayashi, Y., Utsumi, K. (1985) Cyto-prot. Biol 3,143 ; C. A. 107, 211861x.

107 Okajima, K., Koga, S., Inoue, M., Okabe, H., Takatsuki, K. (1987) Igaku no Ayumi 142, 505; C. A. 107, 190669y.

108 Suzuki, M., Kawauchi, H., Fujiyoshi, T., Ueyama, S., Mogi, G. (1987) Arerugi 36, 268; C. A. 107, 168415r.

109 Matsuno, T., Orida, K., Sato, E., Inoue, T., Usumi, K. (1986) Igaku to Yakugaku 16, 445; C. A. 107, 126480c.

${ }^{110}$ Okazaki, T., Suetsugu, M., Tsuji, Y., Kino, A., Yoshida, T. (1987) Jpn. Kokai Tokkyo Koho JP 62, 84, 015; C. A. 107, 46051y.

111 Okazaki, T., Suetsugu, M., Tsuji, Y., Kino, A., Yoshida, T. (1987) Jpn. Kokai Tokkyo Koho JP 62, 84, 013; C. A. 107, 46053a.

112 Okazaki, T., Suetsugu, M., Tsuji, Y., Kino, A., Yoshida, T. (1987) Jpn. Kokai Tokkyo Koho JP 62, 84, 016; C. A. 107, 102438u.

113 Okazaki, T., Suetsugu, M., Tsuji, Y., Kino, A., Yoshida, T. (1987) Jpn. Kokai Tokhyo Koho JP 62, 84, 011; C. A. 107, 46054b.

114 Matsuno, T., Orita, K., Sato, E., Nobori, K., Inoue, B., Utsumi, K. (1987) Biochem. Pharmacol. 36, 1613

115 Sato, T., Kometani, M., Fujii, T. (1987) Thromb. Res. 46, 587; C. A. $107,32933 a$.

116 Uramoto, H., Watanabe, Y., Hagiwara, M., Kikuchi, T., Watanabe, K. (1985) Wakan Iyaku Gakkaishi 2, 246; C. A. 104, 61307 t.

117 Watanabe, H., Uramoto, H., Maeda-Hagaiwara, M., Kikuchi, T. (1985) Arch. Int. Pharmacodyn. Ther. 278, 53.

118 Liu, J., Qiu, P. (1986) Xian Yike Daxue Xuebao 7, 31; C. A. 105 $127038 \mathrm{k}$.

119 Du, Z., Liu, H., Chai, C., Luo, L., Hu, C. (1986) Zhongguo Yaoli Xuebao 7, 419; C. A. 105, 164657g.

120 Zhu, J., Jia, J., Yan, H., Hu, C. (1986) Zhongguo Yaoli Xuebao 7, 543; C. A. 106,12670 p.

121 Su, F., Liu, G., Zhang, X., Guan, X., Guo, L., Hu, C. (1986) Zhongyan Tongbao 11, 692; C. A. 106, 81434b.

122 Zeng, W., Leng, D., Hu, C. (1986) Zhongcayao 17, 497; C. A. 106, $95890 \mathrm{j}$.

123 Fang, D., Jiang, M. (1986) J. Hypertens. 4 (Suppl. 6) 150; C. A. 107, $228891 \mathrm{e}$.

124 Zheng, X., Bian, R. (1986) Zhonguo Yaoli Xuebao 7, 40; C. A. 104, $141957 \mathrm{v}$.

125 Huang, W., Yang, Z., Peng, S. (1987) Yiyao Gongye 18, 447; C. A. $109,6776 y$.

126 Okazaki, T., Suetsugu, M., Tsuji, Y., Kino, A., Yoshida, T. (1987) Jpn. Kokai Tokkyo Koho JP 62, 84, 012; C. A. 107, 102439v.

127 King, V. F., Garcia, M. L., Himmel, D., Reuben, J. P., Lam, Y. T., Pan, J., Han, G., Kaczorowski, G. J. (1988) J. Biol. Chem. 263, 2238.

128 Ogino, T., Sato, S., Sasaki, H., Chin, M. (1987) Jpn. Kokai Tokkyo Koho JP 62, 207, 216; C. A. 109, 11713 e.
129 Zeng, F. D., Shaw, D. H. Jr., Ogilvie, R. I. (1985) J. Cardiovasc. Pharmacol. 7, 1034; C. A. 104, 61770p.

130 Wang, Z., Ma, C., Lin, W., Luo, Z. (1987) Fudan Xuebao Ziran Kexueban 26, 169; C. A. 108, 71584c.

131 Xao, W., Xia, G., Fang, D., Jiang, M. (1987) J. Tongji Med. Univ. 7, 80 ; C. A. $108,87834 \mathrm{v}$.

132 Lai, S., Zhao, T. F., Wang, K. X. (1988) Yaoxue Xuebao 23, 356; C: A. $109,125816 \mathrm{w}$.

133 Seow, W. K., Ferrante, A., Goh, D. B. H., Chalmers, A. H., Li, S., Thong, Y. H. (1988) Int. Arch. Allergy Appl. Immunol. 85, 410; C: A. $108,143058 \mathbf{j}$.

134 Seow, W. K., Ferrante, A., Li, S., Thong, Y. H. (1988) Int. Arch. Allergy Appl. Immunol. 85, 404; C. A. 108, 161238x.

135 Teh, B. S., Seow, W. K., Chalmers, A. H., Playford, S., Ioannoni, B., Thong, Y. H. (1988) Int. Arch. Allergy Appl. Immunol. 86, 220; C. A. $109,433 \mathrm{n}$.

136 Chen, N., Liu, L., Cai, G., Yang, J., Li, Y. (1988) Ecotoxicol. Environ. Saf. 15, 149; C: A. 109, 548d.

137 Liu, L., Chen, N., Cai, G., Li, Z., Yang, J., Li, Y. (1988) Ecotoxicol. Environ. Saf. 15, 142; C. A. 109, 785d.

138 Yao, W., Xia, G., Han, H., Fang, D., Jiang, M. (1986) Zhongguo Yaoli, Xuebao 7, 128; C: A. 104, 161754e.

139 Yu, S., Wang, M., Ke, C., Liu, Y., Cao, L., Gao, Y., Wu, X., Fu, R., Wang, Y. (1986) Zhonghua Yixue Zazhi 66, 29; C. A. 104, 218866 z.

140 Seow, W. K., Li, S. Y., Thong, Y. H. (1986) Immunol. Lett. 13, 83; C: A. $105,108261 \mathrm{~m}$.

141 Xu, Y. (1986) Shengwu Huaxue Zazhi 2, 15; C. A. 106, 12940 b.

${ }^{142}$ Fuska, J. (1985) Chem. Listy 79, 1169; C. A. 104, 84939q.

143 Wang, G., Cheng, B., Zong, X., Fang, D., Jiang, M. (1987) Zhongguo Yaoli Xuebao 8, 522; C: A. 107, 228853u.

144 Zheng, X., Pan, H., Bian, R. (1987) Zhongguo Yaoli Xuebao 8, 525; C. A. $107,228854 \mathrm{v}$.

145 Li, Y., Xu, Y. (1987) Zhongguo Yaoli Xuebao 8, 529; C. A. 107, $229373 z$.

146 Li, W., Zhou, H., Yang, Q., Bian, R. (1987) Zhongguo Yaoli Xuebao 8, 450; C. A. 107,190560 .

147 Zhou, H., Xie, Q., Bian, R. (1987) Yaoxue Xuebao 22, 405; C. A. 107, $168420 \mathrm{p}$.

148 Hu, G., Hu, Y., Fang, D., Jiang, M. (1987) Zhongguo Yaoli Xuebao 8 , 325; C. A. 107, $109100 \mathrm{~m}$.

149 Xu, Y., Ni, J. (1986) Kexue Tongbao 31, 1710; C. A. 107, 54647n.

150 Li, C., Ying, S., Liu, G., Niu, S. (1988) Shandong Yike Daxue Xuebao 26,42 ; C. A. $109,31820 \mathrm{r}$

151 Xu, K., Tan, J., Qiu, C., Zhu, X., Tang, X. (1986) Zhongguo Yaoli Xuebao 7,$422 ;$ C. A. $105,164658 \mathrm{~h}$.

152 Cao, Y., Che, X., Yuan, B. (1986) Yaoxue Xuebao 21, 781; C. A. 106, $43864 \mathrm{j}$.

${ }^{153}$ Xu, K., Tan, J., Ye, X., Qiu, G., Dai, F., Li, X., Zeng, Y. (1987) Yaoxue Xuebao 22, 704; C. A. 107, 228355h.

154 Cao, Y., Che, X., Yuan, B. (1987) Zhongguo Yaloi Xuebao 8, 405; C. A. 107,168719 t.

155 Cao, Y., Che, X. (1987) Yaoxue Xuebao 22, 462; C. A. 107, 168632j.

156 Cao, Y., Che, X. (1987) Xian Yike Daxue Xuebao 8, 38; C. A. 107, $109302 \mathrm{~d}$

157 Bentley, K. W. (1986) Nat. Prod. Rep. 3, 153; C. A. 105, 134196p.

158 Fajardo, V., Podesta, F., Urzua, A. (1986) Rev. Latinoam. Quim. 16 141; C. A. $106,15685 \mathrm{~h}$.

159 Guinaudeau, H., Freyer, A. J., Shamma, M. (1986) Nat. Prod. Rep. 477 ; C. A. 107,96930 e.

${ }^{160}$ Stadler, R., Kutchan, T. M., Loeffler, S., Nagakura, N., Cassels, B., Zenk, M. H. (1987) Tetrahedron Lett. 28, 1251.

161 Herath, W. H. M. W., Hussain, S. F., Freyer, A. J., Guinaudeau, H., Shamma, M. (1987) J. Nat. Prod. 50, 721.

162 Cortes, D., Dadoun, H., Paiva, R. L. R., DeOliveira, A. D. (1987) J. Nat. Prod. 50, 910.

${ }^{163}$ Huang, W., Huang, Z., Yang, Z., Peng, S., Xia, G., Yao, W. (1988) Zhongguo Yaoke Daxue Xuebao 19, 81; C. A. 109, 122015y.

164 Lopez, J. A., Lin, M.-C., Schiff, P. L. Jr. (1988) Phytochemistry 27, 3335.

165 Cassels, B. K., Shamma, M. (1980) Heterocycles 14, 211.

166 Berthou, S., Jossang, A., Guinaudeau, H., Leboeuf, M., Cave, A. (1988) Tetrahedron 44, 2193

167 Guinaudeau, H., Freyer, A. J., Shamma, M., Baser, K. H. C. (1984) Tetrahedron 40, 1975 . 
Ergänzungen bei der Korrektur am 2.3.90

(Literatur vom November 1988 bis Januar 1990)

\section{Neue BBI-Alkaloide}

12-O-Demethyllauberin aus Berberis chilensis (1a); Northalibroline aus Thalictrum minus var. minus (2a); 2-Norisotetrandrine, (+)Stepierine, $(+)-2^{\prime}$-Norobaberine, (+)-Stephibaberine, (+)-2'-Norcepharanthine, (-)2-Norcepharanoline und (-)-Norisocepharanthine aus Stephania pierrii (3a); Dehatridine und Dehatrine (Berbamintyp) aus Dehaasia triandra (4a); 12-O-Demethylcoclobine (Oxyacanthintyp) aus Guatteria guianensis (5a); Berberilaurine (Thalmintyp) aus Berberis laurina (6a); (-)-Cordobine, (-)-Monterine und (-)-Granjine (Rodiasintyp) aus Crematosperma spec. (7a); (+)- $N$-Methyltiliamosine (erstmals als Naturstoff (vergl. 25) aus Tiliacora racemosa (8a).

\section{Synthese}

Totalsynthese von $R, R$-Daurisoline (9a), Darstellung von Synthesevorstufen für Tiliacora-Alkaloide (10a, 11a).

\section{Ergebnisse pharmakologischer Untersuchungen}

Calciumantagonistische Wirkung von BBIAlkaloiden (12a, 13a); Antiarrythmischer Effekt von Neeferine (14a, 15a), Tetrandine (30a); Stimulierende Wirkung von Tetrandrin, Isotetrandrin, Cycleanine und Berbamine auf das Haarwachstum (16a); Effekt von Berbamin auf $\alpha$ Adrenozeptoren (17a, 19a); Schutzwirkung vom Berbamin gegen akute focale cerebrale Ischämie (18a); Inhibitor Effekt von Cepharanthine gegen die Acetylcholinwirkung (20a); Wirkung von Cepharanthin gegen Leukoplakia und Lichen planus (21a); Antimalariawirkung: von BBI-Alkaloiden aus Tiliacora triandra (22a), Tetrandrin (29a); Calciumantagonistische Wirkung von Antioquine (23a); Blutdrucksenkende Wirkung von Fangchinolin-Derivaten (24a); Inhibitor-Wirkung von Cepharanthin auf carcinogene Promoter (25a); Entzündungshemmende und immunsuppressive Wirkung von Tetrandrin $(26 a, 28 a)$ und Berbamin (26a); Bindung von Berbamin und Tetrandrin an Muskarinrezeptoren (27a); Wirkung von Tetrandrin auf die Atmung (31a); Wirkung von 7-O-Demethylisothalicberin auf die Funktion von Herzschrittmachern (32a).

\section{Ergebnisse mit Zellkulturen}

BBI-Alkaloide in Wurzelkulturen von Stephania cepharantha (33a).

\section{Neue botanische Herkunft bekannter BBI-Alkaloide}

Thalrugosin aus Berberis polymorpha (34a); Alkaloide aus Thalictrum minus in Südbulgarien (35a); Alkaloide eines neuen Chemotyps von Thalictrum minus (36a); Alkaloide aus den Wurzeln von Thalictrum glandulosissimum (37a); Berbamin-Alkaloide aus Isopy- rum thalictroides (38a); Alkaloide aus Thalictrum lancesteri (39a); Trilobin-Alkaloide aus den Wurzeln von Anisocycla cymosa (40a).

\section{Übersichtsartikel}

Alkaloids derived from 1-Benzyltetrahydroisoquinoline in some Chilean species of the genera Discaria and Berberis (41a); Structure Studies of BBI-alkaloids (42a).

1a Torres, R., Bol. (1989) Soc. Chil. Quim., 34, 11; C. A. 111, 233323h.

28 Baser, K. H. C., Kirimer, N. (1988) Planta Med. 54, 513.

3a Tantisewie, B., Amurrio, S., Guinaudeau, H., Shamma, M. (1989) J. Nat. Prod. 52, 846 .

4a Lu, S. T., Tsai, I. L., Leou, S. P. (1989) Phytochemistry 28, 615.

5a Berthou, S., Leboeuf, M., Cave, A., Guinaudeau, H. (1989) J. Nat. Prod. 52, 95.

6a Weber, J. F., Le Ray, A. M., Bruneton, J., Fournet, A. (1989) J. Nat. Prod. 52, 81

7a Saez, J., Fernandez, E., Jossang, A., Cave, An., Cave, Ad. (1989) Can. J. Chem. 67, 275

8a Ray, A. K., Mukhopadhyay, G., Mitra, S. K., Guha, K. P., Mukherjee, B., Atta-Ur-Rahman, Nelofar, A. (1989) Phytochemistry, 28, 675.

9a Hua, W., Kong, R. (1988) Zhongguo Yaoke Daxue Xuebao 19, 161 ; C. A. $110,193168 \mathrm{~g}$.

10a Pachaly, P., Schäfer, M. (1989) Arch. Pharm. (Weinheim) 322, 477

11a Pachaly, P., Schäfer, M. (1989) Arch. Pharm. (Weinheim) 322, 483.

12a Hu, Z., Chen, S., Hao, Z., Huang, W., Peng, S., (1988) Cell. Signalling 1,181 ; C. A. 110,147205 u.

13a Cai, H., Huang, Z., Yang, Z., Wang, E., Peng, S. (1989) Zhongguo Yaoke Caxue Xuebao 20, 1 : C. A. 111, $126455 \mathrm{f}$.

14a Li, G., Li, X., Lu, F. (1989) Zhongguo Yaoli Xuebao 10, 328; C. A. $111,108709 \mathrm{n}$

15a Li, G., Li, X., Lu, F. (1989) Zhongguo Yaoli Xuebao 10, 406; C. A. $111,208,873 \mathrm{e}$

$16 a$ Kobayashi, Y., Kodama, H., Nogawa, Y. (1989) Jpn. Kokai Tokkyo Koho JP 0161,413 [89 61, 413], C. A. 111, 120627s.

17a Han, B. Y., Liu, G. Q. (1988) Yaoxue Xuebao, 23, 806; C. A. 111 $126804 u$.

$18 \mathrm{a}$ Bao, L. H., Li, F. L., Li, W. H. (1989) Asia Pac. J. Pharmacol. 4, 163; C. A. $111,208912 \mathrm{~s}$.

19a Liu, G., Han. B., Wang, E. (1989) Zhongguo Yaoli Xuebao 10, 302 ; C. A. 111,108874 .

20a Ishikawa, M., Takayanagi, Y., Sasaki, K. (1988) Annu. Rep. Tohoku Coll. Pharm. 35, 249; C. A. 112, 469r

21a Tsuchiya, M., Inoue, Y., Kurokawa, Y., Imai, K., Yokomizo, M., Tejima, Y., Inoue, N., Mitsuihishi, R. (1989) Byoin Yakugaku 15, 43; C. A. $111,28470 \mathrm{n}$

22a Pavanand, K., Webster, H. K., Yongvanitchit, K., Dechatiwongse, T (1989) Phytother. Res. 3, 215; C. A. 112, 15970w.

23a D'Ocon, M. P., Candenas, M. L., Anselmi, E., Zafra-Polo, M. C. (1989) Arch. Int. Pharmacodyn. Ther. 297, 205; C. A. 110, 165989g.

24a Ogino, S. S., Chin, M., Kawashima, K. (1988) Jpn. Kokai Tokkyo Koho JP 63, 179, 878 [88, 179, 878]; C. A. 110, 29089m.

25a Takido, M., Yasukawa, K., Akasu, M. (1988) Jpn. Kokai Tokkyo Koho JP 63, 179, $826[88,179,826]$; C. A. 110, 121434t

26a Li, S., Ling, L., Teh, B. S., Seow, W. K., Thong, Y. H. (1989) Int. J. Immunopharmacol. 11, 395; C. A. 111, 126472j.

27a Hou, Y. F., Liu, G. Q. (1988) Yaoxue Xuebao 23, 801; C. A. 110, 185334 t.

28a Li, S. Y., Teh, B. S., Seow, W. K., Ling, L. H., Thong, Y. H. (1989) Int. Arch. Allergy Appl. Immunol. 90, 169; C. A. 112, 16027f.

29a Ye, Z., Van Dyke, K. (1989) Biochem. Biophys. Res. Commun. 159, 242; C. A. 110, 170124a.

30a Lou, J., Zhang, C. (1988) Zhonggue Yaoli Xuebao 9, 412; C. A. 109 , $183252 \mathrm{v}$.

31a Castranova, V., Jones, T., Giza, A., Li, C., Li, Y., Frazer, D. (1989) Cotton Dust 13, 125; C. A. 112, 31816f.

32a Moralses, M. A., Gallardo, L. R., Martinez, J. L., Puebla, R. S., Hernandez, D. A. (1989) Gen. Pharmacol. 20, 621; C. A. 111, 224999 f.

33a Sugimoto, Y., Yamada, Y., Sugimura, Y. (1989) J. Nat. Prod. 52, 199. 
34a Urzua, M. A., Espinoza, S. S. (1988) Rev. Latinoam. Quim. 19, 109; C. A. $110,132169 \mathrm{~m}$

35a Dimov, B., Dutschevska, Kh. Kuzmanov, B. (1989) Dokl. Bolg. Akad. Nauk 42, 61; C. A. 111, 211952k.

36a Gasic, O., Durkovic, R., Popovic, M., Pal, B., Dutschevska, H., Georgieva, A. (1989) Fitoterapi8a 60, 382; C. A. 112, 4612 e.

37a Rao, C., Zhang, P., Chen, W., Fang, Q. (1989) Zhongcaoyao 20, 344; C. A. $112,42312 q$.

38a Kostalova, D., Hrochova, V., Uhrin, D., Tomko, J. (1988) Chem. Pap. 42,841 ; C. A. $110,170219 \mathrm{k}$. 39a Lopez, J. A., Lin, M. C., Schiff, P. L. Jr. (1988) Phytochemistry 27, 3335.

40a Kanyinda, B., Diallo, B., Vanhaelen-Fastre (1989) Planta Med. 55, 394.

41a Gaona, R. T. (1988) Contrib. Cient. Tecnol 18, 125; C. A. 110, $132115 r$.

42a Lu, Y. (1989) Shenyang Yaoxuayuan Xuebao 6. 130; C. A. 111, $39638 \mathrm{z}$. 